\title{
The Multi-period Multi-trip Container Drayage Problem with Release and Due Dates
}

\author{
M. Bruglieria ${ }^{a, *}$, S. Mancini ${ }^{\mathrm{b}, \mathrm{d}}$, R. Peruzzini ${ }^{\mathrm{c}}$, O. Pisacane $^{\mathrm{c}}$ \\ ${ }^{a}$ Dipartimento di Design, Politecnico di Milano, Italy \\ ${ }^{b}$ Dipartimento di Matematica e Informatica, Universitá di Cagliari, Italy \\ ${ }^{c}$ Dipartimento di Ingegneria dell'Informazione, Universitá Politecnica delle Marche, Italy \\ ${ }^{d}$ ICELAB, ICT for City Logistics and Enterprises Center, Politecnico di Torino, Italy
}

\begin{abstract}
The Containers Drayage Problem (CDP) aims at routing a fleet of trucks, based at a common terminal, to serve customers, minimizing the total travel distance. Each trip starts from and ends to the terminal, handling a subset of customers. Each customer requires either picking a container up (export customer) or delivering a container (import customer). We introduce a more realistic variant, i.e., the Multi-trip Multi-period CDP with Release and Due Dates (MM-CDP-RDD), in which the planning horizon is composed by several periods (days). On each day, each truck may perform more than one trip respecting the Release and Due Dates (RDD) associated with customer services, corresponding to the first and the last day on which the service can be carried out, respectively. Drivers contracts impose limitations on the maximum time allowed driving on each day, on two consecutive days and on the whole weekly planning horizon. To model the MM-CDP-RDD, we propose both an Arc-based Integer Linear Programming (ILP) formulation and a Trip-based ILP formulation that exploits the generation of all the feasible non-dominated trips. To efficiently address medium/large-sized instances of the problem, we also design six Combinatorial Beneders' Cuts approaches. All the methods are compared on a rich set of instances generated for this new problem.
\end{abstract}

Keywords: Routing, Multi-trip Vehicle Routing, Multi-period Vehicle Routing, Combinatorial Benders' Cuts

\section{Introduction}

In the Port Logistics sector, the term Containers Drayage refers to the goods transportation between terminals (e.g., sea ports, intermodal terminals, inland ports, border points) and customers, where containers are used

\footnotetext{
${ }^{*}$ Corresponding author: maurizio.bruglieri@polimi.it
} 
as bins. The drayage operating cost significantly amounts on the total doorto-door containers transportation cost (Figure 1). For 500-mile haul, it represents about $42 \%$ of the total door-to-door cost [34. This leads to the need of properly routing the trucks used for the drayage operations.

The Containers Drayage Problem (CDP) aims at efficiently routing a fleet of trucks, based at a common terminal, in order to serve customers geographically distributed while minimizing the total travel distance. In a trip, a truck starts from the terminal, serves a subset of customers and returns to the terminal. The customers are distinguished into two categories: import customers who require a container delivery and export customers who instead require a container pickup. Therefore, the drayage activities mainly concern the distribution of trucks that move full/empty containers between the terminal, the import and the export customers [16].

According to the International Standard Organization, several container sizes are permitted (e.g., $10 \mathrm{feet}(\mathrm{ft}), 20 \mathrm{ft}, 40 \mathrm{ft}, 45 \mathrm{ft}, 48 \mathrm{ft}, 53 \mathrm{ft})$, although the most used ones are $20 \mathrm{ft}$ and $40 \mathrm{ft}([37],[42],[27])$ while, the truck capacity is usually equal to $40 \mathrm{ft}$. This means that each truck can transport either one $40 \mathrm{ft}$ sized container or two $20 \mathrm{ft}$ sized containers, simultaneously. Due to these loading restrictions, the maximum number of customers can be served into a single trip is equal to 4 . Figure 2 shows a feasible CDP solution in which 5 trips are performed for serving both export and import customers identified, respectively, by a positive and a negative demand. For instance, a $-40 \mathrm{ft}$ demand means that the customer requires receiving a $40 \mathrm{ft}$ sized container.

However, since the inland destinations are usually not far from the terminal, short-duration trips are very frequent. Therefore, introducing the assumption that a truck can perform more than one trip during a working day is realistic and leads to the multi-trip variant of the CDP ([20], [26], [7]).

The aim of this work is to introduce a new variant of the CDP in which the multi-trip assumption is combined with the need of both serving the customers only in specific periods (e.g, days) and respecting the contractual restrictions imposed on the drivers working time. For this purpose, we divide the whole planning horizon into discrete time periods (e.g., days) and each customer can be served only in specific consecutive periods e.g., Release and Due Dates (RDD). We address simultaneously the problem of assigning containers to the trucks and of routing the latter.

The main contributions of this work are:

- the introduction of the Multi-period Multi-trip (MM) CDP with Relase and Due Dates , i.e., MM-CDP-RDD, a more realistic CDP variant in which the planning horizon is divided into several discrete periods (multi-period), a truck is allowed to perform more than one trip in each period (multi-trip), RDDs are associated with each customer and 


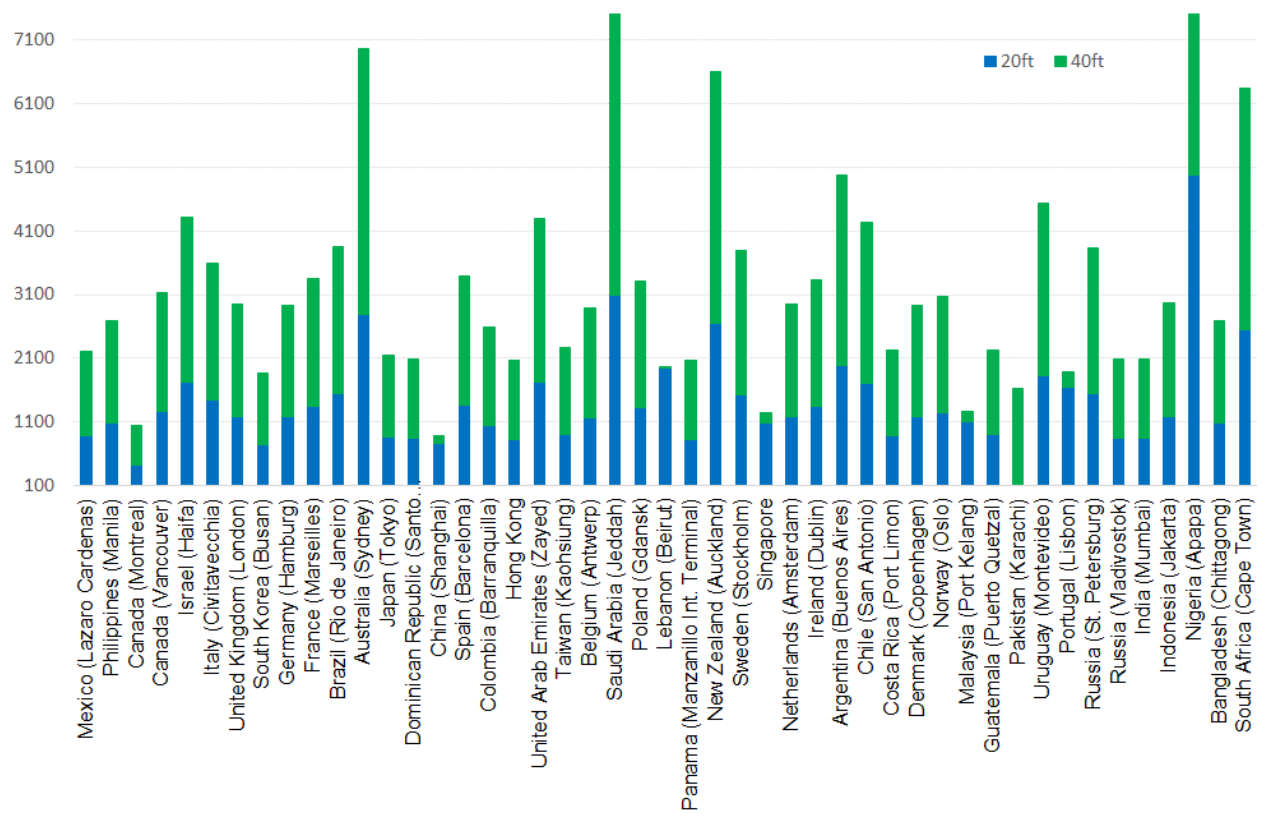

Figure 1: Total costs (in $\$$ ) to move both $20 \mathrm{ft}$ and $40 \mathrm{ft}$ containers from USA to other countries. Source: https://moverdb.com

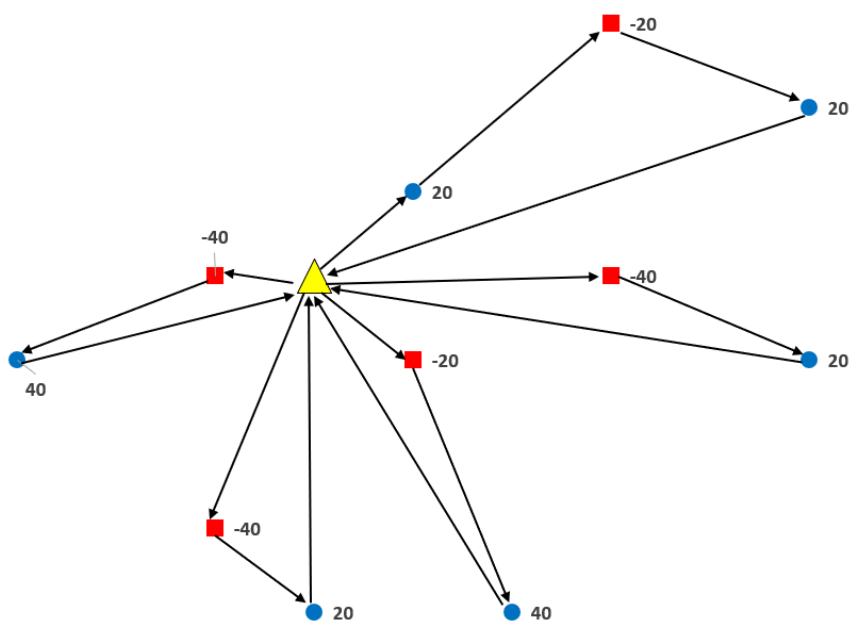

Figure 2: An example of feasible solution of the CDP. 
finally, restrictions on the trip duration in each period, in two consecutive periods and in the whole planning horizon are imposed. The latter assumptions allow accounting for contractual limitations on the drivers' work performance.

- the formulation of an Arc-based Integer Linear Programming (A-ILP) model;

- the design of an exact two-stage approach together with the definition of both trip feasibility and dominance rules. In the first stage, all the feasible non-dominated trips are generated. While, in the second stage, a Trip-based ILP (T-ILP) model is solved;

- the design of several Combinatorial Benders' Cuts (CBC) approaches, based also on valid inequalities ad hoc defined for the MM-CDP-RDD;

- the generation of a rich set of instances for this new problem, starting from the traditional benchmark instances proposed for the Vehicle Routing Problem by both Solomon [36] and Gehring \& Homberger [18];

- the numerical comparisons among A-ILP, T-ILP and the CBC approaches.

The rest of the paper is organized as in the following. Section 2 reviews the main literature contributions on the CDP and its variants. In Section 3 , the statement of the problem is given together with the notation used. In Section 4, the A-ILP model for the MM-CDP-RDD is described, while Section 5 proposes a two-stage approach. Section 6 describes several variants of the $\mathrm{CBC}$ approach specifically designed for the MM-CDP-RDD. In Section 7. benchmark instances for this new problem are generated and numerical comparisons among the solution methods are discussed. Finally, Section 8 draws some conclusions and outlines future research directions worthy of investigation.

\section{Literature Review}

The CDP and its variants belong to the most general class of Vehicle Routing Problem (VRP) with Pickups and Deliveries, i.e., the Pickup and Delivery Problem (PDP) because customers can require either a container pickup or its delivery ([30], [31]). Moreover, as specified by the recent literature review [16] on the CDP and its variants, the scientific contributions can be classified according to the following features: DropEPPick (DP), i.e., trucks and containers can move separately during the drayage operations. In case of delivery requests, this means that the career has not to wait at the customer for picking the empty container up, after the delivery; Stay-with 
(SW), i.e., trucks and containers have to move coupled during the operations; Container per truck, i.e., the number of containers per truck can be either equal 1 or greater than 1; Time Windows, i.e., trucks have to perform the service at customers within specific time windows and this usually complicates the solution of the problem.

In this paper, we introduce the following two new features: Multi-period (MP), i.e., the planning horizon is divided into discrete time periods (e.g., days) and then, the release and due dates at customers are specified in terms of periods in which they have to be served; Multi-trip (MT), i.e., each truck can perform more than one trip in each period. We discuss the main literature contributions on $\mathrm{CDP}$ and its variants with regard the aforementioned features.

The Drop\&Pick CDP with Time Windows (TW) at customers, with only one container per truck, has been extensively studied in the literature. In [21], the CDP with intermediate facilities between terminals and customers in a metropolitan area is addressed and modeled as a multi-Traveling Salesman Problem with TW (m-TSPTW) at both origins and destinations. An exact solution approach based on dynamic programming is proposed. Since it is able to solve to optimality only instances with up to $15-20$ nodes, a heuristic approach that combines it with a genetic algorithm is also proposed to address instances with up to 100 nodes. In [14, the problem is modeled as a Multi-Resource Routing Problem with flexible tasks for determining the set of routes by resource type, satisfying all the tasks, respecting operational rules. For it, a set partitioning model is formulated with a weighted objective function minimizing both the fleet and the variable distance cost and a greedy randomized procedure is also designed. While, a cluster method together with a reactive Tabu Search (TS) is proposed in [50]. In [49], the problem is addressed as a m-TSPTW and solved by a modified version of the method of [46]. While, in [51], it is modelled as a m-TSPTW with resource constraints and solved through a reactive TS. Considering that, for each empty container, either the origin or the destination is not defined, in [5], the problem is modelled as an asymmetric m-TSPTW and both a sequential and an integrated solution approaches are described. For minimizing the total operating time of all the trucks used, in [39], a node-arc mathematical formulation is proposed, for simultaneously routing the trucks, scheduling the services and relocating the empty containers while, a TS is proposed for solving large-sized instances. In [45], a variant of the problem is studied in which, with 4 types of containers (inbound/outbound, full/empty), the transport can be performed by both trucks and trains and a node-arc mathematical formulation with a hybrid TS are proposed. Assuming multidepots and a homogeneous fleet, in [29], a node-arc mathematical model is formulated for minimizing the total operating time and a two-stage heuristic is also designed. Allowing that a tractor can be assigned to a different trailer to perform a new task, in [48], a node-arc formulation is modelled 
coordinating the empty containers that move between customers. A TS algorithm is also designed for solving instances with up to 400 customers while a maxmin ant colony optimization algorithm is proposed in [47].

A problem variant in which the intermodal terminal requires trucks to have an appointment is addressed in [34]. Then, an arc-node formulation together with a reactive TS algorithm are proposed. Finally, assuming that some empty containers can return to the depot for maintenance, in [38], the problem is seen as an extension of the asymmetric VRP with TW and an arcnode formulation is solved through a branch-and-price-and-cut algorithm. A Drop\&Pick CDP with TW at customers with more than one container per truck is addressed in [43. where the problem is modelled as a multiple matching problem to determine the optimal pickup and delivery vehicle routes and a variable neighborhood search is also proposed.

A Drop\&Pick CDP without TW at the customers, with only one container per truck, is addressed in [6], modelled as an asymmetric multiple vehicle TSP considering that either the origin or the destination of the empty containers are unknown in advance, minimizing simultaneously the number of vehicles used and the total travel distance. Both a hybrid deterministic annealing algorithm and a TS are designed.

A Stay-with CDP with only one container per truck is addressed by [28] where a first phase model is formulated for identifying the maximum revenue set of container move tasks to serve and a second phase routing and scheduling model is solved. In addition, the authors also design a column generation based heuristic. With more than one container per truck, the problem is modelled as a VRP with multiple visits and heterogeneous trucks in [23] and a variant of the Clarke-and-Wright algorithm is also proposed. While, an Adaptive Guidance meta-heuristic is proposed in [22].

The Stay-with CDP with TW at customers is addressed in [9], where the problem is solved through a local search, based on three neighbourhoods, with an initial solution computed by a two-phase insertion heuristic. Several techniques already proposed in the literature for the VRPs are extended in order to schedule pre- and end-haulage of containers in [32] and several versions are studied, i.e., both managing multiple empty container depots and balancing empty container depot levels. In [35], the problem is modelled through Mixed Integer quadratic programming in order to schedule simultaneously tractor, loaded container, empty container and chassis and a reactive TS is also developed.

In the CDP proposed in [33] for taking under control the harmful emissions, collaboration among truckers is permitted and a mathematical model based on the m-TSPTW is formulated. Finally, in [16], the authors, after introducing the possibility to move more than one container per truck in a trip and by exploiting the limited number of feasible trips, propose a set-covering formulation tested on real-world case studies.

Multi-trip CDPs are addressed in both [26] and [7], the latter considers 
Table 1: Literature contributions on CDP and its variants

\begin{tabular}{|c|c|c|c|c|c|c|c|}
\hline Reference & Service & Container/truck & RDD & TW & MP & MT & Approach \\
\hline 46 & $\mathrm{DP}$ & 1 & & $\checkmark$ & & & $\mathrm{NA}+\mathrm{heu}$ \\
\hline 21 & $\mathrm{DP}$ & 1 & & $\checkmark$ & & & NA+heu \\
\hline 14 & DP & 1 & & $\checkmark$ & & & $\mathrm{T}+$ heu \\
\hline 50 & DP & 1 & & $\checkmark$ & & & $\mathrm{NA}+$ heu \\
\hline 49 & $\mathrm{DP}$ & 1 & & $\checkmark$ & & & $\mathrm{NA}+$ heu \\
\hline 51 & $\mathrm{DP}$ & 1 & & $\checkmark$ & & & NA+heu \\
\hline 5 & DP & 1 & & $\checkmark$ & & & $\mathrm{NA}+$ heu \\
\hline 39 & $\mathrm{DP}$ & 1 & & $\checkmark$ & & & NA+heu \\
\hline 45 & $\overline{\mathrm{DP}}$ & 1 & & $\checkmark$ & & & $\mathrm{NA}+$ heu \\
\hline 29 & $\mathrm{DP}$ & 1 & & $\checkmark$ & & & $\mathrm{NA}+$ heu \\
\hline 48 & DP & 1 & & $\checkmark$ & & & $\mathrm{NA}+$ heu \\
\hline 47 & DP & 1 & & $\checkmark$ & & & $\mathrm{NA}+$ exact \\
\hline 34 & $\mathrm{DP}$ & 1 & & $\checkmark$ & & & NA+heu \\
\hline 38 & $\mathrm{DP}$ & 1 & & $\checkmark$ & & & $\mathrm{T}+$ exact \\
\hline 11 & DP & $\geq 1$ & & $\checkmark$ & & & $\mathrm{NA}+$ heu \\
\hline 43 & DP & $\geq 1$ & & $\checkmark$ & & & M+heu \\
\hline 6 & $\overline{\mathrm{DP}}$ & 1 & & & & & NA+heu \\
\hline 44 & $\mathrm{DP}$ & $\geq 1$ & & & & & M+heu \\
\hline 28 & SW & 1 & & & & & T+heu \\
\hline 23 & SW & $\geq 1$ & & & & & NA+heu \\
\hline 22 & SW & $\geq 1$ & & & & & NA+heu \\
\hline 20 & SW & 1 & & & & $\checkmark$ & $\mathrm{NA}+\mathrm{heu}$ \\
\hline 17 & SW & 1 & & $\checkmark$ & & & NA+heu \\
\hline 9 & SW & 1 & & $\checkmark$ & & & $\mathrm{NA}+\mathrm{heu}$ \\
\hline 32 & SW & 1 & & $\checkmark$ & & & $\mathrm{T}+$ exact \\
\hline 35 & SW & 1 & & $\checkmark$ & & & $\mathrm{NA}+$ heu \\
\hline 33 & SW & 1 & & $\checkmark$ & & & $\mathrm{NA}+$ exact \\
\hline 16 & SW & $\geq 1$ & & $\checkmark$ & & & $\mathrm{T}+$ exact \\
\hline 26 & DP & 1 & & $\checkmark$ & & $\checkmark$ & T+heu \\
\hline 7 & SW & $\geq 1$ & & $\checkmark$ & & $\checkmark$ & $\mathrm{NA}+$ exact \\
\hline This work & DP & $\geq 1$ & $\checkmark$ & & $\checkmark$ & $\checkmark$ & $\mathrm{NA}+\mathrm{T}+$ exact \\
\hline
\end{tabular}

only $20 \mathrm{ft}$ sized containers. However, neither of them considers multiple periods. Therefore, to the best of our knowledge, no contributions already exist in which the multi-period is combined with the multi-trip, the RDDs of the customers, the contractual restrictions on the trip duration, allowing both moving more than one container per truck and managing two container sizes (20ft and 40ft).

Table 1 summarizes the most significant contributions on the CDPs, where column Container/truck specifies the number of containers can be simultaneously moved by each truck. While, $N A, T$ and $M$ identify respectively the Node-Arc, the Trip-based and the Matching formulation. Finally, heu and exact denote respectively that heuristic and exact approaches have been developed.

\section{Problem statement and notation}

The MM-CDP-RDD aims at efficiently routing a fleet of $K$ trucks, based at a common terminal 0 , for serving import/export customers (set $C$ ), minimizing the total travel distance over a given planning horizon. For this 
Table 2: Notation of the MM-CDP-DRD

\begin{tabular}{|c|c|}
\hline Set & Meaning \\
\hline$C$ & Set of customers \\
$T$ & Set of trucks \\
$T_{i}$ & Set of periods \\
$T_{i j}$ & Set of periods in which both customer $i$ and customer $j$ can be served \\
$C_{\pi}$ & Set of customers served by the trip $\pi$ \\
$\tilde{T}_{\pi}$ & Set of periods in which the trip $\pi$ can be performed \\
\hline \hline Parameter & Meaning \\
\hline 0 & Terminal \\
$Q$ & Average truck speed \\
$T_{1}^{\text {max }}$ & Loading truck capacity \\
$T_{2}^{\text {max }}$ & Maximum travel time per truck per period \\
$T_{3}^{\text {max }}$ & Maximum travel time per truck in two consecutive periods \\
$d_{i j}$ & Maximum travel time per truck in the planning horizon \\
$r_{i}$ & Travel distance between $i \in C \cup\{0\}$ and $j \in C \cup\{0\}$ \\
$\tau_{i}$ & Demand of customer $i$ \\
$d_{\pi}$ & Service time at customer $i$ \\
$a_{i \pi}$ & Total distance travelled in the trip $\pi$ \\
\hline
\end{tabular}

purpose, the planning horizon is divided into $T$ discrete time periods, each one representing a working day. Hereafter, we suppose $T=\{1,2, \ldots,|T|\}$. Within the same period, each truck can perform more than one trip. However, due to drivers contractual limitations, each truck can travel no longer than $T_{1}^{\max }, T_{2}^{\max }$ and $T_{3}^{\max }$, in a period, in two consecutive periods and in the whole planning horizon (generally, a week), respectively, 24. Each truck, whose average speed is equal to $v$, has also a limited loading capacity $Q$ equal to $40 \mathrm{ft}$ meaning that it can simultaneously move either only one container of $40 \mathrm{ft}$ or two containers of $20 \mathrm{ft}$.

The demand $r_{i}$ of each customer $i \in C$ can be either positive (export customer) or negative (import customer) and of either $40 \mathrm{ft}$ or $20 \mathrm{ft}$. While, the service time at each customer $i \in C$ is indicated by $\tau_{i}$. In addition, for each customer $i \in C$, the set $T_{i}$ indicates the set of consecutive periods on which the service can be performed at customer $i$. Moreover, for each pair $(i, j): i, j \in C, T_{i j}$ indicates the subset of $T$ containing the periods in which both customer $i$ and customer $j$ can be served, i.e., $T_{i j}=T_{i} \cap T_{j}$. For each pair $(i, j) \in C \cup\{0\}$, the travel distance $d_{i j}$ is known. Table 2 summarizes the notation used in this paper.

A trip $\pi$ starts from the terminal and returns to it, by serving a sub-set of customers $C_{\pi}$. Its total distance is indicated by $d_{\pi}$ and the periods in which it can be performed is denoted by $\tilde{T}_{\pi}=\cap_{i \in C_{\pi}} T_{i}$. Finally, we also introduce a customers coverage matrix in which the entry $a_{i \pi}$ is 1 if and only if the customer $i \in C$ is served in the trip $\pi$, i.e., $i$ belongs to $C_{\pi} ; 0$, otherwise.

Since each truck can move either a $40 \mathrm{ft}$ container or two containers of $20 \mathrm{ft}$, all possible kinds of feasible trips can be enumerated according to the fol- 
lowing definition.

\section{Definition 1: Type of trips}

- 1-customer trip $\{0, i, 0\}$, with $r_{i} \in\{40,-40,20,-20\}$;

- 2-customer trip $\{0, i, j, 0\}$, with $\left(r_{i}, r_{j}\right) \in\{(-20,-20),(20,20),(20,-20)$, $(-20,20),(-40,20),(-20,40),(-40,40)\}$;

- 3-customer trip $\{0, i, j, k, 0\}$, where $\left(r_{i}, r_{j}, r_{k}\right) \in\{(-20,-20,20),(-20,20,20)$, $(-20,20,-20),(20,-20,20),(-20,-20,40),(-40,20,20)\}$;

- 4-customer trip $\{0, i, j, k, v, 0\}$, where $\left(r_{i}, r_{j}, r_{k}, r_{v}\right) \in\{(-20,20,-20,20)$, $(-20,-20,20,20)\}$.

\section{An Arc-based Mathematical Programming Formulation}

Similarly to the traditional VRPs, the MM-CDP-DRD can be formally represented on a directed graph $G=(N, A)$, where the set of nodes $N$ contains the set $C$ of customers to be served and the terminal 0 while, $A$ is the set of the arcs.

According to Definition 1, $A$ is not complete because, through a proper pre-processing, only some arcs are kept. More specifically, $A=\{(i, j) \in$ $C \times C: i \neq j \wedge\left(r_{i}, r_{j}\right) \in\{(-20,-20),(20,20),(20,-20),(-20,20),(-40,20),(-$ $\left.20,40),(-40,40)\} \wedge T_{i} \cap T_{j} \neq \emptyset \wedge d_{i j} / v \leq T_{1}^{\max }\right\} \cup\left\{(0, j), j \in C: d_{0 j} / v \leq\right.$ $\left.T_{1}^{\max }\right\} \cup\left\{(j, 0), j \in C: d_{j 0} / v \leq T_{1}^{\max }\right\}$. Figure 3 shows the graph $G$ generated for the instance of the problem of Figure 2 .

The formulation is based on the following decision variables: $x_{i j}^{k t}$, binary variable equal to 1 if truck $k$ travels from node $i$ to node $j$ in period $t$ and 0 otherwise, $\forall(i, j) \in A, \forall k \in K$ and $\forall t \in T ; u_{i}^{+}$, an integer variable denoting the total quantity of pickup until node $i$ from the last exit from $0, \forall i \in N$; $u_{i}^{-}$, an integer variable representing the total quantity of delivery until node $i$ from the last exit from $0, \forall i \in N$.

The Arc-based Integer Linear Programming (A-ILP) formulation of the MM-CDP-DRD is the following:

$$
\min \sum_{(i, j) \in A} \sum_{k \in K} \sum_{t \in T_{i j}} d_{i j} x_{i j}^{k t}
$$

s.t.

$$
\begin{gathered}
\sum_{k \in K} \sum_{j:(i, j) \in A} \sum_{t \in T_{i j}} x_{i j}^{k t}=1 \quad \forall i \in C \\
\sum_{\substack{j:(i, j) \in A \\
t \in T_{j}}} x_{i j}^{k t}-\sum_{\substack{j:(j, i) \in A \\
t \in T_{j}}} x_{j i}^{k t}=0 \quad \forall i \in N, k \in K, t \in T_{i} \\
\sum_{\substack{(i, j) \in A \\
t \in T_{i j}}}\left(\frac{d_{i j}}{v}+2 \tau_{j}\right) x_{i j}^{k t} \leq T_{1}^{\max } \quad \forall k \in K, t \in T
\end{gathered}
$$




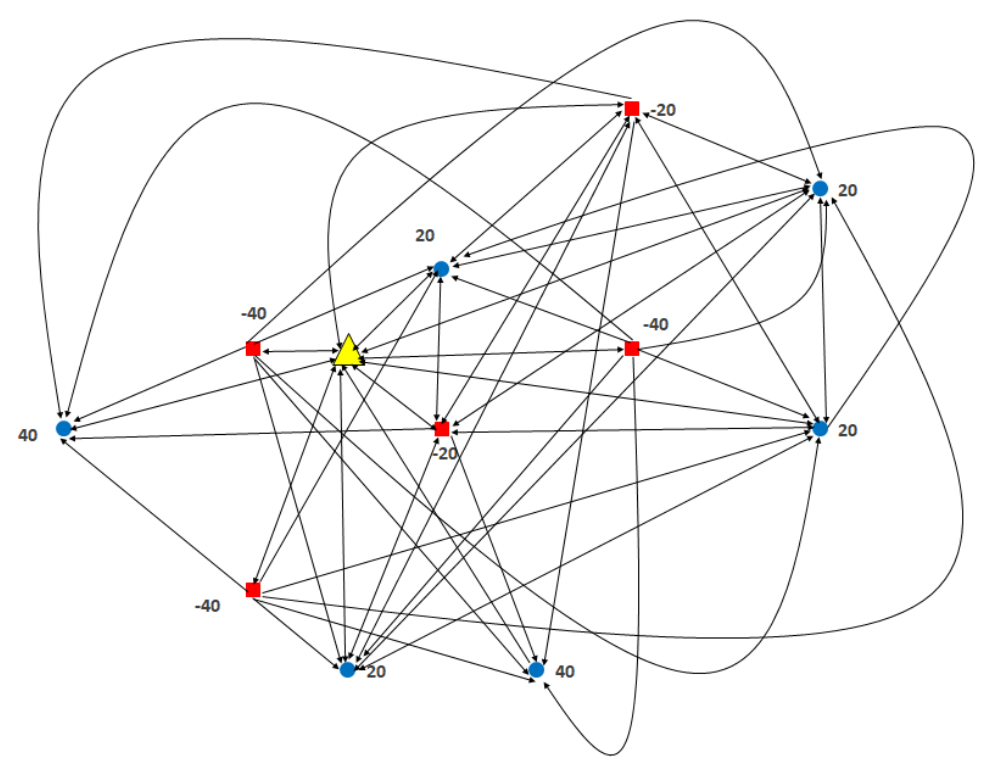

Figure 3: A graph instance of the MM-CDP-RDD.

$\sum_{\substack{(i, j) \in A \\ t \wedge(t+1) \in T_{i j}}}\left(\frac{d_{i j}}{v}+2 \tau_{j}\right) x_{i j}^{k t}+\sum_{\substack{(i, j) \in A \\ t \wedge(t+1) \in T_{i j}}}\left(\frac{d_{i j}}{v}+2 \tau_{j}\right) x_{i j}^{k(t+1)} \leq T_{2}^{\max } \quad \forall k \in K, t=1, \ldots,|T|-1$

$$
\sum_{(i, j) \in A} \sum_{t \in T_{i j}}\left(\frac{d_{i j}}{v}+2 \tau_{j}\right) x_{i j}^{k t} \leq T_{3}^{\max } \quad \forall k \in K
$$

$$
u_{0}^{+}=u_{0}^{-}=0
$$

$$
\begin{gathered}
u_{j}^{+} \geq u_{i}^{+}+\max \left\{r_{j}, 0\right\}-\left(Q+\max \left\{r_{j}, 0\right\}\right)\left(1-\sum_{k \in K} \sum_{t \in T_{i j}} x_{i j}^{k t}\right) \quad \forall(i, j) \in A: j \neq 0 \\
u_{j}^{-} \geq u_{i}^{-}-\min \left\{r_{j}, 0\right\}-\left(Q-\min \left\{r_{j}, 0\right\}\right)\left(1-\sum_{k \in K} \sum_{t \in T_{i j}} x_{i j}^{k t}\right) \quad \forall(i, j) \in A: j \neq 0 \\
x_{i j}^{k t} \leq u_{i}^{-}-u_{i}^{+} \quad \forall(i, j) \in A: i \neq 0, j \neq 0, r_{i}=r_{j}=-20, \forall k \in K, \forall t \in T_{i j} \\
x_{i j}^{k t} \leq Q-u_{i}^{+} \quad \forall(i, j) \in A: i \neq 0, j \neq 0, r_{i}=20, r_{j}=-20, \forall k \in K, \forall t \in T_{i j} \\
u_{i}^{-} \leq Q \quad \forall i \in C \\
u_{i}^{+} \leq Q \quad \forall i \in C
\end{gathered}
$$




$$
\begin{gathered}
\sum_{k \in K} \sum_{t \in T_{i}} x_{i 0}^{k t}=1 \quad \forall i \in C: r_{i}=+40 \\
\sum_{k \in K} \sum_{t \in T_{i}} x_{0 i}^{k t}=1 \quad \forall i \in C: r_{i}=-40 \\
x_{j 0}^{k t} \geq x_{i j}^{k t} \quad \forall k \in K, i \in C, j \in C: i \neq j, r_{i}=r_{j}=20, t \in T_{i j} \\
x_{i j}^{k t} \in\{0,1\} \quad \forall(i, j) \in A, k \in K, t \in T_{i j} \\
u_{i}^{+}, u_{i}^{-} \geq 0 \text { integer } \quad \forall i \in C
\end{gathered}
$$

The objective function (1) to be minimized represents the total travel distance. Constraints (2) assure that each customer is served exactly once while constraints (3) guarantee the flow conservation for each node of the graph. Constraints (4)-(6) assure that each truck cannot travel longer than $T_{1}^{\max }, T_{2}^{\max }$ and $T_{3}^{\max }$, respectively, in a period, in two consecutive periods and in the whole planning horizon. It is worth noting that the service time of each customer is counted twice for taking into account the related service time at the terminal.

Constraint (7) fixes to 0 the quantity of both pickup and delivery at the terminal while, constraints (8)-(9) count the quantity of pickup and delivery at each node, respectively. Since, these constraints are imposed for each arc except those entering in the terminal, they also ensure that the subtours not visiting the terminal cannot be feasible.

Constraints (10) ensure that two consecutive deliveries of $20 \mathrm{ft}$ size cannot be served after a pickup of $20 \mathrm{ft}$ size, i.e., a trip $\{0, v, i, j, 0\}$, where $r_{v}=$ $20, r_{i}=r_{j}=-20$, is not allowed. Similarly, constraints (11) avoid that a pickup and a delivery, both of $20 \mathrm{ft}$ size, are consecutively served after a delivery and a pickup, both of $20 \mathrm{ft}$ size, i.e., a trip $\{0, q, v, i, j, 0\}$, where $r_{q}=r_{j}=-20, r_{v}=r_{i}=20$, is not permitted.

Constraints (12)-(13) guarantee that the maximum truck loading capacity is never exceeded. In a trip, a customer $i \in C$ with $r_{i}=40 \mathrm{ft}$ has to be served as the first (14) while each customer $i \in C$ with $r_{i}=-40 \mathrm{ft}$ has to be served as the last (15). A truck, after consecutively visiting two customers $i$ and $j$ with $r_{i}=r_{j}=20 \mathrm{ft}$, has to return to the terminal (16). Finally, constraints (17)-(18) define the decision variables nature.

\section{A two-stage trip-based approach}

In this section, the two-stage trip-based approach designed for solving the MM-CDP-RDD is described, by exploiting the generation rules (Definition 1). The approach proposed works as in the following. A trip generation procedure is firstly invoked (Section 5.1). In order to further reduce the number of trips to manage, both feasibility and dominance rules are also 
introduced. This way, the set $\Pi$ of all feasible non-dominated trips is generated. Then, a Trip-based Integer Linear Programming formulation (T-ILP) is solved receiving $\Pi$ as input (Section 5.2 ).

\subsection{Trip generator}

From all the possible trips generated according to the rules introduced in Section 3 , only the feasible are kept, according to the following definition:

\section{Definition 2: Feasible trip.}

A trip $\pi$ is feasible if and only if:

- $t_{\pi}=\frac{d_{\pi}}{v} \leq T_{1}^{\max }$;

- $\tilde{T}_{\pi}=\bigcap_{i \in C_{\pi}} T_{i} \neq \emptyset$;

- The truck capacity $Q$ is never exceeded.

Moreover, in order to reduce as much as possible the number of trips to manage, among the feasible ones, we maintain only the set $\Pi$ of nondominated ones, according to the following definition:

Definition 3: Dominated trip.

Given two feasible trips $\pi_{1}, \pi_{2}$, the former dominates the latter if and only if:

- $C_{\pi_{1}} \equiv C_{\pi_{2}}$

- $d_{\pi_{1}}<d_{\pi_{2}}$;

In Algorithm 1, the trips generation procedure is outlined. In particular, the algorithm starts generating the 1 -customer feasible trips. It is worth noting that all of them are non-dominated too. The routine Feasible $(\pi)$ returns TRUE if the trip $\pi$ is feasible according to the feasibility rules (Definition 2); FALSE, otherwise. Then, for each feasible 1-customer trips, it generates all the feasible non-dominated 2 -customer trips. In particular, a 2 -customer trip $\{0, c 1, c 2,0\}$ is obtained by the feasible 1 -customer trip $\{0, c 1,0\}$ by adding $c 2: c 2 \neq c 1$ as the last served. This way, a 3 -customer trip $\{0, c 1, c 2, c 3,0\}$ is obtained by the feasible non-dominated 2-customer trip $\{0, c 1, c 2,0\}$ by adding $c 3: c 3 \neq c 1 \wedge c 3 \neq c 2$ as the last served. Moreover, a 4 -customer trip $\{0, c 1, c 2, c 3, c 4,0\}$ is obtained by the feasible non-dominated 3 -customer trip $\{0, c 1, c 2, c 3,0\}$ by adding $c 4$ such that $c_{4} \notin\left\{c_{1}, c_{2}, c_{3}\right\}$.

Generating 2-customer, 3-customer and 4-customer trips means checking not only the feasibility conditions but also the dominance ones. In fact, the routine Dominated $(\pi)$ returns TRUE if the trip $\pi$ is dominated, according to the dominance rules (Definition 3); FALSE, otherwise. 


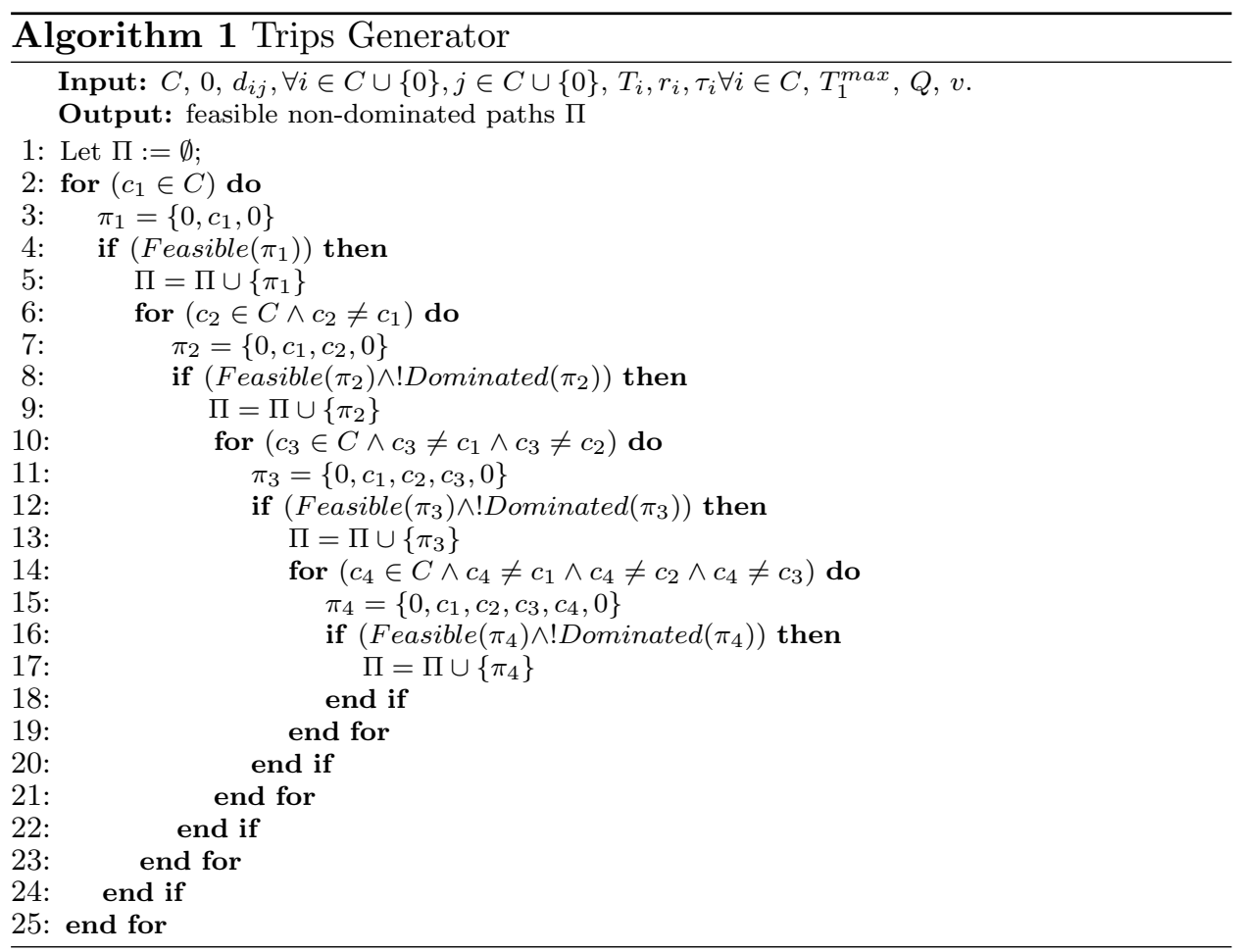

\subsection{Trip-based Integer Linear Programming model}

The Trip-based ILP model (T-ILP) receives in input the set $\Pi$ of all feasible non-dominated trips, generated through Algorithm 1. It is formulated by introducing the following decision variables: $z_{\pi}$ equal to 1 if trip $\pi \in \Pi$ is selected; 0 otherwise; $y_{\pi k}^{t}$ equal to 1 if trip $\pi \in \Pi$ is performed by truck $k \in K$ on period $t \in \tilde{T}_{\pi}$.

The mathematical formulation is given in what follows.

$$
\begin{gathered}
\min \sum_{\pi \in \Pi} d_{\pi} z_{\pi} \\
\sum_{\pi \in \Pi} a_{i \pi} z_{\pi}=1 \quad \forall i \in C \\
\sum_{k \in K} \sum_{t \in \tilde{T}_{\pi}} y_{\pi k}^{t}=z_{\pi} \quad \forall \pi \in \Pi \\
\sum_{\pi \in \Pi: t \in \tilde{T}_{\pi}} t_{\pi} y_{\pi k}^{t} \leq T_{1}^{\max } \quad \forall k \in K, \forall t \in T \\
\sum_{\pi \in \Pi: t \in \tilde{T}_{\pi}} t_{\pi} y_{\pi k}^{t}+\sum_{\pi \in \Pi: t \in \tilde{T}_{\pi}} t_{\pi} y_{\pi k}^{t+1} \leq T_{2}^{\max } \quad \forall k \in K, \forall t=1, \ldots,|T|-1 \\
\sum_{t \in T} \sum_{\pi \in \Pi: t \in \tilde{T}_{\pi}} t_{\pi} y_{\pi k}^{t} \leq T_{3}^{\max } \quad \forall k \in K \\
z_{\pi} \in\{0,1\} \quad \forall \pi \in \Pi
\end{gathered}
$$




$$
y_{\pi k}^{t} \in\{0,1\} \quad \forall \pi \in \Pi, \forall k \in K, \forall t \in T
$$

The objective function 19 to be minimized represents the total travel distance. Customers' coverage is guaranteed by constraints (20) while constraints (21) logically link variables $y$ and $z$, i.e., a trip $\pi$ is selected if and only if it is assigned at a truck in a period. Constraints (22)-(24) assure that a truck cannot travel longer than $T_{1}^{\max }, T_{2}^{\max }$ and $T_{3}^{\max }$, respectively, in a period, in two consecutive periods and in the whole planning horizon. Finally, constraints (25)-(26) define the variables nature.

\section{Combinatorial Beneders' Cuts approaches}

Benders' Decomposition (BD) is a successful and broadly applied exact approach to solve Mixed Integer Programming (MIP) models introduced in the pioneering work of Benders [4]. The core idea, which this approach is based on, consists in fixing a set of variables, which make the MIP hard to be solved, so that the problem can be strongly simplified. In the BD, the problem is decomposed into a Master Problem (MP), in which only a subset of the decision variables is considered and into a Slave Problem (SP), containing the remaining variables. The MP and the SP are iteratively solved in sequence.

At each iteration, the MP is firstly solved, then, the SP is formulated by fixing the variables values found by the MP, and solved for the remaining variables. Based on the SP outcome, one or more cuts can be generated and added to MP, preventing it from exploring specific areas of the search space. In the classical application of BD to MIPs, the SP is a Linear Programming (LP) problem, whose dual solution is exploited to derive cuts to be added to the MP. In [15], BD has been generalized and extended to problems in which the SP is not required to be an LP problem.

Logic Based Benders decomposition, introduced many decades later in [19], is an extension of the classical BD in which, in the MP only the variables that contribute directly to the objective function are considered, while, the others are relegated to the SP. This way, the SP becomes a pure feasibility problem. Each time the SP results to be infeasible, one or more cuts can be generated to cut off infeasible solutions from the MP solutions search space. As soon as a feasible SP is detected, the overall solution obtained is proved to be optimal.

A specific case of Logic Based Benders decomposition, named Combinatorial Benders decomposition, has been presented in [12]. This approach is specifically suited for MIP models, involving binary variables and a large number of logical implications through Big-M constraints. In this scenario, each time a given combination of the MP variables yields to an infeasible SP, a Combinatorial Benders Cut (CBC) is added, forcing to be 0 at least one of the variables equal to 1 in the current $\mathrm{MP}$ optimal solution. If the number 
of variables equal to 1 in the MP optimal solution is large, the derived cut may be very weak. Stronger cuts can be obtained identifying a subset of variables responsible for the infeasibility through the search of the Minimum Infeasible Set (MIS), which can be determined through either an exact or a heuristic approach. Such cuts are stronger since they allow cutting off from the MP search space several solutions at a time, i.e., all the variables contained in the MIS equal to 1, strongly speeding-up the convergence toward an optimal solution. However, the algorithm convergence is always guaranteed even adopting only standard CBC, cutting off one solution at a time as proved in [12].

In the last decade, CBC has been successfully applied to real problems arising in different contexts. The first application is described in [3], where a toll facilities location problem is addressed. In [13, an exact CBC-based approach for the Strip Packing problem is proposed. Several applications in the field of Port Logistics are reported in the literature, e.g., quayside operations at container terminals are studied in [8] and [10, while in [41], the lock scheduling problem is addressed. Other innovative applications can be found in health care, regarding beam intensity modulation in radiotherapy [40], in production, where the assembly line balancing is studied [1] and in jobs allocation on computers clusters, [25].

All the above cited problems share a common structure where the variables can be partitioned into two subsets. The first one, involved in the MP, contains all the variables directly contributing to the objective function, while the second one, containing variables only responsible for the solution feasibility, is involved in the SP.

In this section, we propose several CBC approaches in which we try to speed-up the standard CBC method both providing stronger cuts and adding valid inequalities to the MP.

\section{1. $C B C 1$}

The first developed $\mathrm{CBC}$ approach $(\mathrm{CBC} 1)$ is based on the classical framework of [12]. The master problem, MP1, aims at finding the optimal subset of trips covering all the customers at the minimum cost and, therefore it becomes a pure Set Partitioning problem, quickly solved to optimality.

MP1:

$$
\begin{gathered}
\min \sum_{\pi \in \Pi} d_{\pi} z_{\pi} \\
\sum_{\pi \in \Pi} a_{i \pi} z_{\pi}=1 \quad \forall i \in C \\
z_{\pi} \in\{0,1\} \quad \forall \pi \in \Pi
\end{gathered}
$$

The objective function (27) to be minimized represents the total travel distance as in the T-ILP model, while constraints (28) and (29) correspond to constraints (20) and 25), respectively. 
The slave problem, SP1, checks if a feasible assignment of those trips to both periods and trucks exists such that the duration constraints are respected, becoming a pure feasibility problem.

SP1:

$$
\begin{gathered}
\sum_{k \in K} \sum_{t \in \tilde{T}_{\pi}} y_{\pi k}^{t}=z_{\pi}^{\star} \quad \forall \pi \in \Pi \\
\sum_{\pi \in \Pi: t \in \tilde{T}_{\pi}} t_{\pi} y_{\pi k}^{t} \leq T_{1}^{\text {max }} \quad \forall k \in K, \forall t \in T \\
\sum_{\pi \in \Pi: t \in \tilde{T}_{\pi}} t_{\pi} y_{\pi k}^{t}+\sum_{\pi \in \Pi: t \in \tilde{T}_{\pi}} t_{\pi} y_{\pi k}^{t+1} \leq T_{2}^{\max } \quad \forall k \in K, \forall t=1, \ldots,|T|-1 \\
\sum_{t \in T} \sum_{\pi \in \Pi: t \in \tilde{T}_{\pi}} t_{\pi} y_{\pi k}^{t} \leq T_{3}^{\max } \quad \forall k \in K \\
y_{\pi k}^{t} \in\{0,1\} \quad \forall \pi \in \Pi, \forall k \in K, \forall t \in T
\end{gathered}
$$

where $z_{\pi}^{\star}$ is equal to 1 if trip $\pi$ has been selected in the MP1 optimal solution; 0 otherwise. Constraints (30) imply that if a trip has been selected by MP1, it must be assigned to exactly one period and one truck, while constraints (30)-(34) correspond to (21)-(26) of the T-ILP model.

MP1 and SP1 are iteratively solved in sequence. If SP1 is infeasible, then a cut is added to MP1, imposing that at least one of the trip selected by MP1 would not be selected in the next iterations:

$$
\sum_{\pi \in \Pi^{\star}} z_{\pi} \leq\left|\Pi^{\star}\right|-1
$$

where $\Pi^{\star}$ is the set of the trip selected by MP1 at the current iteration and $\left|\Pi^{\star}\right|$ indicates the number of trips belonging to the set $\Pi^{\star}$.

The procedure is repeated until SP1 becomes feasible. As soon as a feasible solution for SP1 is found, it is proved to be optimal.

The main drawback of $\mathrm{CBC} 1$ is that if the number of trips selected by MP1 is very large, the cuts may become too weak, and, therefore, the convergence toward an optimal solution may result to be slow.

\section{2. $C B C 2$}

In the second $\mathrm{CBC}$ approach proposed, $(\mathrm{CBC} 2)$, we focus our attention on the identification of a subset of the selected trips which is responsible for the SP infeasibility. In particular, we focus on the selected trips which can be performed only on a given subset of consecutive periods $S$ and whose duration imposes that they cannot be paired among them, thus requiring a truck each one. The number of selected trips of this type cannot be greater than the number of trucks multiplied by the number of periods in $S$, to have a feasible solution. 
Both the MP and the SP, namely MP2 and SP2, respectively, exactly correspond to MP1 and SP1. Each time SP2 turns out to be infeasible, for all the periods in $S$, we check if the number of the selected trips, that can be performed only on those periods and cannot be paired with others, is greater than $|S| \cdot|K|$. If this happens, we add to MP2, beyond the traditional cut (35), the following cut:

$$
\sum_{\pi \in \Pi_{S}^{\star}} z_{\pi} \leq|S| \cdot|K|
$$

where $\Pi_{S}^{\star}$ represents the set of trips selected by MP2, that can be performed only on periods belonging to $S$ and cannot be paired among them. The cut (36) becomes very useful when trips are reasonably long respect to the maximum truck usage duration per period, $T_{1}^{\max }$ and when the set of periods in which a customer can be servedis smaller than the total number of periods, $|T|$, e.g., when the number trips $\pi$ for which $\left|\tilde{T}_{\pi}\right|=1$ is high. In fact, the smaller $|S|$, the stronger the related cut is.

\section{3. $C B C 3$}

The third $\mathrm{CBC}$ approach designed, (CBC3), follows the same core idea of $\mathrm{CBC} 2$. However, instead of dynamically adding constraints on the maximum number of non-combinable trips per period, whenever this restriction is violated by the current MP, now, these constraints are directly imposed in the MP from the beginning, acting as valid inequalities. Therefore, MP3 can be obtained adding to MP1 the following valid inequalities:

$$
\sum_{\pi \in \Pi_{S}} z_{\pi} \leq|S| \cdot|K| \quad \forall S \subset T
$$

where $S$ is every subset of consecutive periods selected over $T$ and $\Pi_{S}$ is the set of all the trips that can be performed only on periods belonging to $S$ and cannot be paired among them. While, SP3 exactly corresponds to SP1.

It is worth noting that the total number of valid inequalities (37) only increases in quadratic way in function of $|T|$, since the total number of subsets $S$ of consecutive periods, selected over $T$, is $|T|-|S|+1$ and $|S|$ can vary from 1 to $|T|$.

\section{4. $C B C 4$}

In the fourth $\mathrm{CBC}$ approach, $(\mathrm{CBC} 4)$, we pursue a complete different philosophy to identify stronger cuts. MP4 and SP4 exactly correspond to MP1 and SP1, respectively. The main difference is that, whenever SP4 turns out to be infeasible, i.e., no feasible solution exists with all the $\left|\Pi^{\star}\right|$ selected by the MP, we try to compute the smallest integer value $\alpha^{*}$ for which a feasible solution can be obtained containing at most $\left|\Pi^{\star}\right|-\alpha^{*}$ trips. This approach is able to find very strong cuts and, consequently, to reduce 
the number of iterations needed to reach optimality. However, the computational time of each iteration may become slightly higher than that required by the other CBC approaches. It is worth noting that the greater the value of $\alpha^{*}$, the stronger the cut, but the longer the computational time required to solve the feasibility check problem.

More in details, MP4 and SP4 are iteratively solved in sequence. Whenever SP4 turns out to be infeasible, a Feasibility Check Problem, (FCP4), is formulated. FCP4 corresponds to SP4 except for the fact that constraints (28) are replaced by the following ones:

$$
\begin{gathered}
\sum_{k \in K} \sum_{t \in \tilde{T}_{\pi}} y_{\pi k}^{t}=z_{\pi} \quad \forall \pi \in \Pi \\
\sum_{\pi \in \Pi^{\star}} z_{\pi} \leq\left|\Pi^{\star}\right|-\alpha
\end{gathered}
$$

with $\alpha$ initially equal to 2 . While FCP4 remains infeasible, we increase $\alpha$ by 1 and reiterate until FCP 4 turns out to be feasible. In this way, we exactly identify the maximum number of trips, $\alpha^{\star}=\left|\Pi^{\star}\right|-\alpha$, among those selected by MP4, that can be selected in a feasible solution. Therefore, we can replace the classical CBC (35), to be added to the MP, by the following stronger cut:

$$
\sum_{\pi \in \Pi^{\star}} z_{\pi} \leq \alpha^{\star}
$$

The CBC4 pseudo-code is in Algorithm 2 .

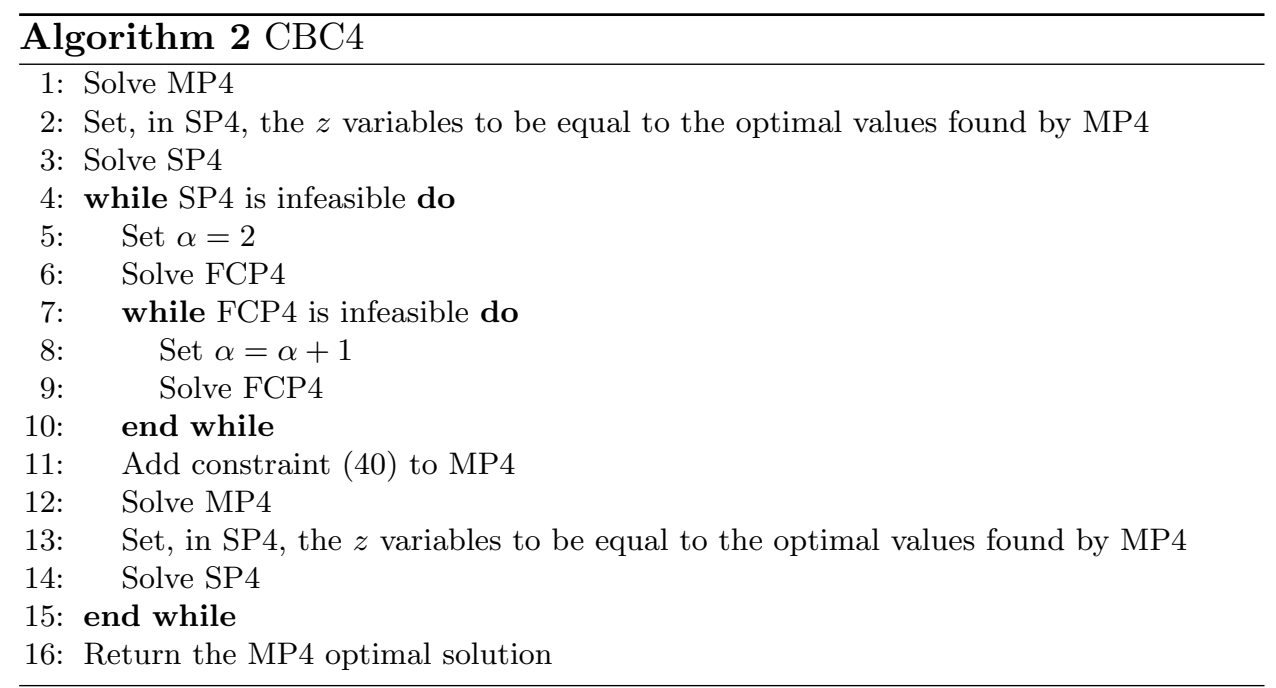

\section{5. $C B C 5$}

The fifth CBC approach, (CBC5), follows a similar idea of CBC2. However, instead of working on the maximum number of non-combinable trips, 
that can be performed only on a given period $t$, which can be selected by the MP, it deals with the maximum total duration of the selected trips that can be performed only on a given period $t$. We then formulate two Feasibility Check Problem whenever the slave problem is infeasible. The first one, $F C P_{5}^{1}$, checks, for each period $t$, if the total duration of the trips, that can be performed only on $t$, selected by MP 5 (indicated by $\Pi_{t}^{\star}$ ), does not exceed $T_{1}^{m a x}$, multiplied by the number of trucks, $|K|$. The same consideration is applied for each pair of consecutive periods $t, t+1$ in the second Feasibility Check Problem, $\left(F C P_{5}^{2}\right)$. In fact, the total duration of the trips, that can be performed only on the subset of periods $t, t+1$, selected by MP5 (indicated by $\left.\Pi_{t, t+1}^{\star}\right)$, has not to exceed the maximum allowed duration $T_{2}^{\max }$, multiplied by $|K|$. If $F C P 5^{1}$ turns out to be infeasible, the following cut is added, together with the classical CBC, (35):

$$
\sum_{\pi \in \Pi_{t}^{\star}} z_{\pi} \leq\left|\Pi_{t}^{\star}\right|-1 \quad \forall t \in T
$$

while if $F C P 5^{2}$ turns out to be infeasible, the following cut is added, together with the classical CBC, (35):

$$
\sum_{\pi \in \Pi_{t, t+1}^{\star}} z_{\pi} \leq\left|\Pi_{t, t+1}^{\star}\right|-1 \quad \forall t=1, \ldots,|T|-1
$$

The CBC5 pseudo-code is in Algorithm 3 .

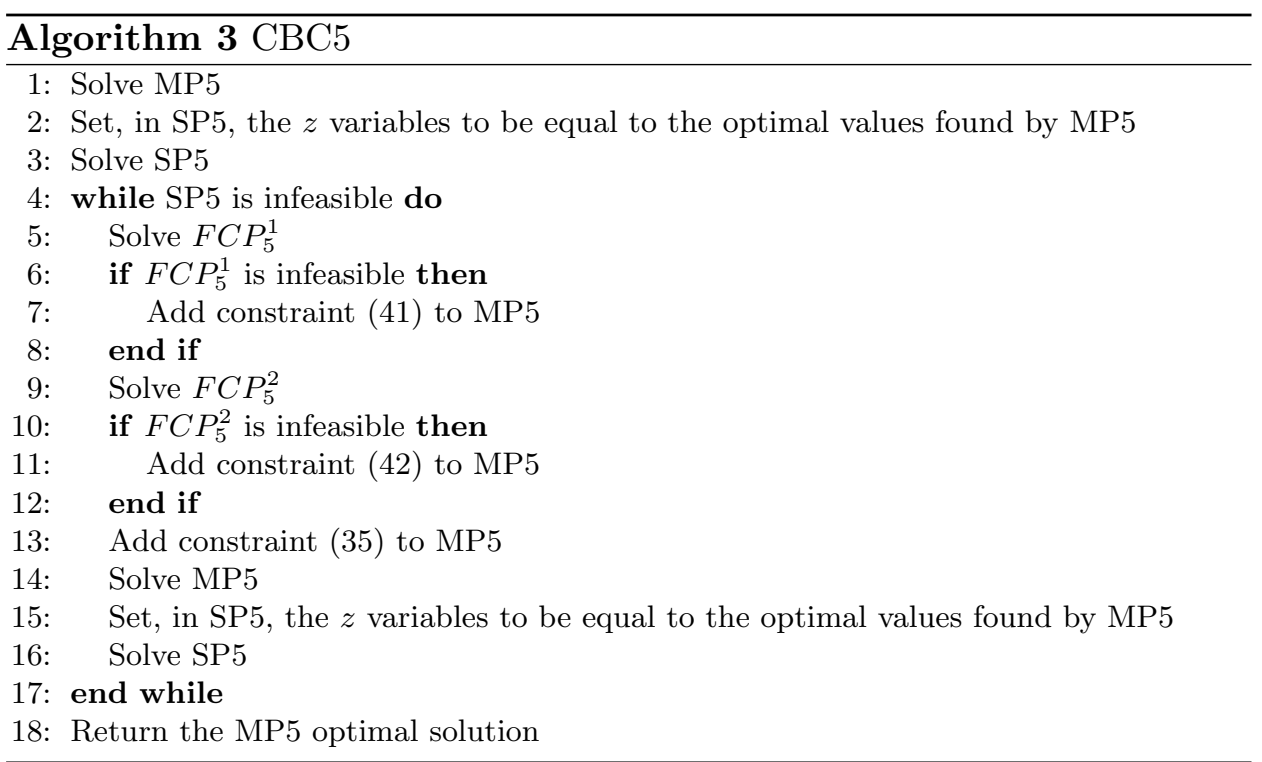

\section{6. $C B C 6$}

Finally, the sixth CBC approach, CBC6, is based on the same idea of CBC5 but all the cuts (41) and (42) are directly added to the MP, at the 
beginning of the search process. According to this, MP6 results to be equal to MP1 with the additional constraints:

$$
\begin{gathered}
\sum_{\pi \in \Pi_{t}} t_{\pi} z_{\pi} \leq|K| \cdot T_{1}^{\max } \quad \forall t \in T \\
\sum_{\pi \in \Pi_{t, t+1}} t_{\pi} z_{\pi} \leq|K| \cdot T_{2}^{\max } \quad \forall t=1, \ldots,|T|-1
\end{gathered}
$$

where $\Pi_{t, t+1}$ indicates the set of trips that can be performed only on the pairs of periods $(t, t+1)$. SP6 exactly corresponds to SP1. Both CBC6 and CBC5 are particularly useful when $\left|\tilde{T}_{\pi}\right|$ is small, but differently from what happens in $\mathrm{CBC} 2$ and $\mathrm{CBC} 3$, they are effective even when trips are short with respect to $T_{1}^{\max }$, i.e., when the number of trips which can be performed by the same truck on a period is high.

\section{Computational results}

In this section, we describe the experimental campaign carried out on several small/medium/large-sized instances, ad hoc generated for the MMCDP-RDD. In particular, in Section 7.1. the procedure for generating the sets of instances is detailed while in Section 7.2, numerical comparisons among the proposed CBC approaches and the two ILP formulations are discussed. All the proposed approaches were implemented in Java (in Eclipse environment) and both the ILP models were solved by ILOGs CPLEX Concert Technology (version 12.9). The experiments were run on a computer with a 64 -bit operating system, $2.39 \mathrm{GHz}$ processor and $32 \mathrm{~GB}$ of RAM.

\subsection{Problem instances generation and parameters setting}

In order to generate significant instances for the MM-CDP-RDD, the dataset proposed for the VRP, available at https://www.sintef.no/projectweb/ top/vrptw/, was considered. In particular, we used the instance sets with 25 and 100 customers proposed by Solomon and the one with 200 customers of Gehring \& Homberger.

A planning horizon of one week, excluding Sunday, was considered, i.e., $|T|=6$. The number of trucks available may change instance per instance. In fact, for each instance, we run the approach with different fleet sizes in order to determine its minimum value to make the instance feasible. Then, we set the fleet size for that instance to the minimum value found. The parameters $T_{1}^{\max }, T_{2}^{\max }$ and $T_{3}^{\max }$ were set to 11,16 and 40 hours, respectively. The truck loading capacity and the truck average speed were set to $40 \mathrm{ft}$ and $60 \mathrm{~km} / \mathrm{h}$. The service times for $20 \mathrm{ft}$ and $40 \mathrm{ft}$ sized containers were set to 15 and 30 minutes, respectively.

For generating the RDDs at customers, the procedure proposed in [2] was used. Initializing $t$ equal to 1 , the release date of a customer was set to $t$. The $t$ value was increased by 1 every time a new customer was considered until it 
reached $|T|$ (customers are considered sequentially). After which, $t$ was set again to 1 . The procedure ends when all customers have been considered. Instead, the due date of a customer was determined as the release date plus an integer parameter $\lambda$ that can vary in $[0,2]$. For feasibility reasons, if the release date of customer $i$ is 6 , then $T_{i}=\{6\}$ while if the release date of customer $j$ is 5 , with $\lambda=2$, then $T_{j}=\{5,6\}$.

The distance between each pair of customers and between each customer and the terminal were computed using the Euclidean formulas, considering the coordinates indicated in the original files.

The demand of each customer was set as in the following. We firstly fixed the probability $\beta$ of having a 40ft sized demand and the probability $\gamma$ of having a pickup request. Therefore, for each customer, two integer numbers, $n^{\prime}$ and $n^{\prime \prime}$, were randomly generated with uniform probability, both in $[0,1]$. If, $n^{\prime} \leq \beta$, then the request size was set to $40 \mathrm{ft}$; otherwise, to $20 \mathrm{ft}$. Moreover, if $n^{\prime \prime} \leq \gamma$, the customer request was a pickup; otherwise, a delivery.

Finally, for each of the original six sets with 25,100 and 200 customers, we took randomly one instance. This way, by varying $\lambda$ in $\{0,1,2\}, \beta$ in $\{0.25,0.50,0.75\}$ and $\gamma$ in $\{0.25,0.50,0.75\}$, we generated 162 instances with 25, 100 and 200 customers, for a total number of 486 instances.

\subsection{Result comparisons}

In this section, we compare the results obtained by the proposed CBC approaches and the ones detected by both A-ILP and T-ILP. In the tables that will follow, instance denotes the instance name. We point out that we keep the original instance name in the root name, adding information about parameters $\beta, \gamma$ and $\lambda$, after it. For example, $c 107 \_25 \_25 \_0$ means that the instance $c 107$ has been elaborated with $\beta=0.25, \gamma=0.25$ and finally, $\lambda=0$. Column $|K|$ indicates the minimum number of trucks to make the instance feasible. Columns $T D$ and $C P U$ denote the total travel distance and the CPU time required, respectively. Finally, column $G A P(\%)$ reports the percentage ILP GAP of the ILP models and the symbol $\dagger$ is always used for marking the cases in which the CPU time limit of one hour is reached

\subsubsection{Results on instances with 25 customers}

In this section, we discuss the results obtained by A-ILP and T-ILP on the instances with 25 customers. We do not report the results obtained by the CBC approaches since, due to the very small size of the instances, T-ILP shows on the average the best performance.

Tables 344 show that T-ILP is suitable to close to optimality all the instances in an average total CPU time of 0.60 seconds against 1,005.25 seconds required by A-ILP. It is worth remarking that the total CPU time 
required by T-ILP counts also the time for generating all the feasible nondominated trips. The number of trips generated is equal to 174 in about 0.11 seconds, on average.

Moreover, A-ILP reaches the CPU time limit of 3,600 seconds on four instances that correspond to cases in which the value of $\lambda$ has been set to the maximum one (i.e., 2). Indeed, on these four instances, although A-ILP finds the optimal solution, the solver is not able to certificate its optimality within the CPU time limit. In fact, those percentage gaps are high only because the A-ILP bound turns out to be not tight enough, as often it occurs for arc-based mathematical programming formulations.

\subsubsection{Results on instances with 100 customers}

In this section, the results obtained by the two ILP models are compared with those found by the CBC approaches, on the instances with 100 customers. In particular, the average number of trips generated is almost equal to 19,143 in an average CPU time of about 1.25 seconds, while, the number of cuts added is about 11 in $\mathrm{CBC} 1, \mathrm{CBC} 2$ and $\mathrm{CBC} 3,1$ in $\mathrm{CBC} 4$ and CBC6 and finally, 4 in CBC5.

Tables 5 5 show that on average CBC6 outperforms all the other approaches and the two ILP models, solving all the instances to optimality in the smallest average CPU time of 8.13 seconds. The other CBC approaches instead do not close to optimality two instances (i.e., c106_25_50_2 and $\left.r 207 \_25 \_50 \_2\right)$, cases in which the values of the objective function $(2,517.37$ and $2,195.69$, respectively) are lowest than the optimal ones $(2,521.93$ and $2,199.11$, respectively) because they correspond to the last infeasible solutions found by the MP. Indeed, in the tables, these cases correspond to ones in which the CBC approaches reach the CPU time limit of one hour. Moreover, CBC4 does not close to optimality also the instances $c 106 \_50 \_50 \_2$, $r$ 109_50_50_2, $r 207 \_50 \_50 \_2$ and $r c 108 \_25 \_75 \_2$. This is justified by the fact that, at each iteration, it needs checking not only the feasibility of the current solution found by MP4 but also, finding $\alpha^{*}$.

However, observing the results instance by instance, we can conclude that there is not an approach that always dominates the others. Figure 4 shows the percentage of instances in which each approach dominates the others. Instead, the A-ILP model closes to optimality only $12.96 \%$ of instances while, for the $28.40 \%$ of instances, the solver is not able to certificate the optimality in 1 hour. Finally, on the $58.64 \%$ of instances, the A-ILP model is not suitable to find even a feasible solution in 1 hour (cases in which TD value is indicated by NFS, i.e., No Feasible Solution, in the tables).

\subsection{Results on instances with 200 customers}

In this section, we discuss a comparison between the results obtained by T-ILP model and those of CBC6 that, among the other CBC approaches, shows the best performances on the instances with 100 customers. 
Table 3: Results on instances with 25 customers: Part I

\begin{tabular}{|c|c|c|c|c|c|c|c|}
\hline & & & & & & & \\
\hline$\overline{\text { Instance }}$ & $\mid \overline{|K|}$ & $\overline{T D}$ & $\overline{\mathrm{CPU}}$ & GAP(\%) & $\overline{\mathrm{TD}}$ & $\overline{\mathrm{CPU}}$ & GAP(\%) \\
\hline$c 107 \_25 \_25 \_0$ & 1 & 768.24 & 0.14 & 0.00 & 768.24 & 0.56 & 0.00 \\
\hline c107_25_25_1 & 1 & 595.29 & 32.34 & 0.00 & 595.29 & 0.64 & 0.00 \\
\hline c107_25_25_2 & 1 & 559.47 & $\dagger$ & 8.80 & 559.47 & 0.77 & 0.00 \\
\hline c107_25_50_0 & 1 & 898.05 & 0.13 & 0.00 & 898.05 & 0.39 & 0.00 \\
\hline c107_25_50_1 & 1 & 659.93 & 5.50 & 0.00 & 659.93 & 0.56 & 0.00 \\
\hline c107_25_50_2 & 1 & 634.78 & 14.08 & 0.00 & 634.78 & 0.75 & 0.00 \\
\hline$c 107 \_25 \_75 \_0$ & 1 & 792.76 & 0.25 & 0.00 & 792.76 & 0.61 & 0.00 \\
\hline$c 107 \_25 \_75 \_1$ & 1 & 603.53 & 204.35 & 0.00 & 603.53 & 1.41 & 0.00 \\
\hline$c 107 \_25 \_75 \_2$ & 1 & 539.03 & 1844.99 & 0.00 & 539.03 & 1.48 & 0.00 \\
\hline$c 107 \_50 \_25 \_0$ & 1 & 908.66 & 0.14 & 0.00 & 908.66 & 0.38 & 0.00 \\
\hline c107_50_25_1 & 1 & 761.44 & 1.08 & 0.00 & 761.44 & 0.42 & 0.00 \\
\hline c107_50_25_2 & 1 & 725.07 & 11.51 & 0.00 & 725.07 & 0.59 & 0.00 \\
\hline c107_50_50_0 & 1 & 794.07 & 0.11 & 0.00 & 794.07 & 0.41 & 0.00 \\
\hline c107_50_50_1 & 1 & 586.36 & 0.38 & 0.00 & 586.36 & 0.86 & 0.00 \\
\hline c107_50_50_2 & 1 & 557.80 & 8.92 & 0.00 & 557.80 & 0.77 & 0.00 \\
\hline c107_50_75_0 & 1 & 873.61 & 0.09 & 0.00 & 873.61 & 0.36 & 0.00 \\
\hline c107_50_75_1 & 1 & 810.24 & 0.69 & 0.00 & 810.24 & 0.47 & 0.00 \\
\hline c107_50_75_2 & 1 & 794.02 & 12.22 & 0.00 & 794.02 & 1.13 & 0.00 \\
\hline c107_75_25_0 & 1 & 914.85 & 0.09 & 0.00 & 914.85 & 0.34 & 0.00 \\
\hline$c 107-75 \_25 \_1$ & 1 & 858.66 & 0.14 & 0.00 & 858.66 & 0.39 & 0.00 \\
\hline$c 107 \_75 \_25 \_2$ & 1 & 788.36 & 0.50 & 0.00 & 788.36 & 0.59 & 0.00 \\
\hline c107_75_50_0 & 1 & 883.23 & 0.09 & 0.00 & 883.23 & 0.34 & 0.00 \\
\hline$c 107-75 \_50 \_1$ & 1 & 701.40 & 0.17 & 0.00 & 701.40 & 0.38 & 0.00 \\
\hline c107_75_50_2 & 1 & 661.46 & 0.27 & 0.00 & 661.46 & 0.45 & 0.00 \\
\hline c107_75_75_0 & 2 & 994.82 & 0.09 & 0.00 & 994.82 & 0.37 & 0.00 \\
\hline c107_75_75_1 & 1 & 891.64 & 0.13 & 0.00 & 891.64 & 0.42 & 0.00 \\
\hline c107_75_75_2 & 1 & 831.63 & 0.17 & 0.00 & 831.63 & 0.44 & 0.00 \\
\hline c204_25_25_0 & 1 & 856.01 & 0.17 & 0.00 & 856.01 & 0.36 & 0.00 \\
\hline$c 204 \_25 \_25 \_1$ & 1 & 738.82 & 129.41 & 0.00 & 738.82 & 0.61 & 0.00 \\
\hline c204_25_25_2 & 1 & 682.12 & 3091.02 & 0.00 & 682.12 & 0.88 & 0.00 \\
\hline c204_25_50_0 & 1 & 883.80 & 0.13 & 0.00 & 883.80 & 0.61 & 0.00 \\
\hline$c 204 \_25 \_50 \_1$ & 1 & 746.62 & 109.45 & 0.00 & 746.62 & 1.06 & 0.00 \\
\hline c204_25_50_2 & 1 & 714.51 & $\dagger$ & 12.55 & 714.51 & 1.00 & 0.00 \\
\hline c204_25_75_0 & 1 & 999.95 & 1.33 & 0.00 & 999.95 & 0.59 & 0.00 \\
\hline c204_25_75_1 & 1 & 814.05 & 1.22 & 0.00 & 814.05 & 0.55 & 0.00 \\
\hline$c 204 \_25 \_75 \_2$ & 1 & 795.20 & 19.09 & 0.00 & 795.20 & 1.36 & 0.00 \\
\hline$c 204 \_50 \_25 \_0$ & 1 & 1016.70 & 0.09 & 0.00 & 1016.70 & 0.34 & 0.00 \\
\hline c204_50_25_1 & 1 & 1014.47 & 0.33 & 0.00 & 1014.47 & 0.55 & 0.00 \\
\hline c204_50_25_2 & 1 & 982.30 & 2.70 & 0.00 & 982.30 & 0.44 & 0.00 \\
\hline c204_50_50_0 & 1 & 886.50 & 0.14 & 0.00 & 886.50 & 0.34 & 0.00 \\
\hline c204_50_50_1 & 1 & 710.02 & 0.61 & 0.00 & 710.02 & 0.50 & 0.00 \\
\hline$c 204 \_50 \_50 \_2$ & 1 & 648.42 & 8.08 & 0.00 & 648.42 & 0.89 & 0.00 \\
\hline$c 204 \_50 \_75 \_0$ & 1 & 893.21 & 0.22 & 0.00 & 893.21 & 0.39 & 0.00 \\
\hline$c 204 \_50 \_75 \_1$ & 1 & 724.24 & 9.59 & 0.00 & 724.24 & 0.88 & 0.00 \\
\hline$c 204 \_50 \_75 \_2$ & 1 & 686.19 & 107.57 & 0.00 & 686.19 & 0.69 & 0.00 \\
\hline c204_75_25_0 & 1 & 991.22 & 0.09 & 0.00 & 991.22 & 0.36 & 0.00 \\
\hline$c 204 \_75 \_25 \_1$ & 1 & 877.94 & 0.14 & 0.00 & 877.94 & 0.47 & 0.00 \\
\hline$c 204 \_75 \_25 \_2$ & 1 & 853.63 & 0.58 & 0.00 & 853.63 & 0.55 & 0.00 \\
\hline c204_75_50_0 & 1 & 1026.18 & 0.11 & 0.00 & 1026.18 & 0.42 & 0.00 \\
\hline c204_75_50_1 & 1 & 876.06 & 0.16 & 0.00 & 876.06 & 0.38 & 0.00 \\
\hline$c 204 \_75 \_50 \_2$ & 1 & 814.15 & 0.19 & 0.00 & 814.15 & 0.55 & 0.00 \\
\hline$c 204 \_75 \_75 \_0$ & 2 & 1083.95 & 0.11 & 0.00 & 1083.95 & 0.41 & 0.00 \\
\hline$c 204 \_75 \_75 \_1$ & 1 & 922.15 & 0.13 & 0.00 & 922.15 & 0.36 & 0.00 \\
\hline$c 204 \_75 \_75 \_2$ & 1 & 906.29 & 0.28 & 0.00 & 906.29 & 0.45 & 0.00 \\
\hline$r 101 \_25 \_25 \_0$ & 1 & 989.49 & 0.13 & 0.00 & 989.49 & 0.41 & 0.00 \\
\hline$r 101 \_25 \_25 \_1$ & 1 & 855.85 & 2.86 & 0.00 & 855.85 & 0.72 & 0.00 \\
\hline$r 101 \_25 \_25 \_2$ & 1 & 809.65 & 24.48 & 0.00 & 809.65 & 0.84 & 0.00 \\
\hline$r$ 101_25_50_0 & 1 & 942.06 & 0.16 & 0.00 & 942.06 & 0.39 & 0.00 \\
\hline$r$ 101_25_50_1 & 1 & 747.08 & 5.28 & 0.00 & 747.08 & 0.69 & 0.00 \\
\hline$r 101 \_25 \_50 \_2$ & 1 & 696.45 & 98.79 & 0.00 & 696.45 & 1.61 & 0.00 \\
\hline$r 101 \_25 \_75 \_0$ & 1 & 1019.41 & 0.13 & 0.00 & 1019.41 & 0.38 & 0.00 \\
\hline$r$ 101_25_75_1 & 1 & 813.72 & 4.55 & 0.00 & 813.72 & 0.72 & 0.00 \\
\hline$r$ 101_25_75_2 & 1 & 766.84 & 85.82 & 0.00 & 766.84 & 0.94 & 0.00 \\
\hline$r$ 101_50_25_0 & 1 & 981.95 & 0.11 & 0.00 & 981.95 & 0.39 & 0.00 \\
\hline$r 101 \_50 \_25 \_1$ & 1 & 800.29 & 0.69 & 0.00 & 800.29 & 0.89 & 0.00 \\
\hline$r 101 \_50 \_25 \_2$ & 1 & 789.48 & 10.81 & 0.00 & 789.48 & 0.84 & 0.00 \\
\hline r101_50_50_0 & 1 & 1038.76 & 0.13 & 0.00 & 1038.76 & 0.36 & 0.00 \\
\hline$r$ 101_50_50_1 & 1 & 864.63 & 0.34 & 0.00 & 864.63 & 0.55 & 0.00 \\
\hline$r$ 101_50_50_2 & 1 & 806.89 & 1.30 & 0.00 & 806.89 & 0.78 & 0.00 \\
\hline$r 101 \_50 \_75 \_0$ & 1 & 1069.61 & 0.13 & 0.00 & 1069.61 & 0.37 & 0.00 \\
\hline$r 101 \_50 \_75 \_1$ & 1 & 939.93 & 0.30 & 0.00 & 939.93 & 0.50 & 0.00 \\
\hline$r 101 \_50 \_75 \_2$ & 1 & 907.59 & 1.61 & 0.00 & 907.59 & 1.22 & 0.00 \\
\hline$r$ 101_75_25_0 & 1 & 1119.24 & 0.08 & 0.00 & 1119.24 & 0.39 & 0.00 \\
\hline$r 101 \_75 \_25 \_1$ & 1 & 1070.84 & 0.13 & 0.00 & 1070.84 & 0.64 & 0.00 \\
\hline$r 101 \_75 \_25 \_2$ & 1 & 1034.65 & 0.30 & 0.00 & 1034.65 & 0.70 & 0.00 \\
\hline$r 101$ 755_50_0 & 1 & 1093.76 & 0.09 & 0.00 & 1093.76 & 0.34 & 0.00 \\
\hline$r$ 101_75_50_1 & 1 & 969.64 & 0.17 & 0.00 & 969.64 & 0.39 & 0.00 \\
\hline$r 101 \_75 \_50 \_2$ & 1 & 909.40 & 0.30 & 0.00 & 909.40 & 0.70 & 0.00 \\
\hline$r 101$ 75_75_0 & 2 & 1064.31 & 0.17 & 0.00 & 1064.31 & 0.41 & 0.00 \\
\hline$r 101 \_75 \_75 \_1$ & 1 & 951.95 & 0.19 & 0.00 & 951.95 & 0.48 & 0.00 \\
\hline$r 101 \_75 \_75 \_2$ & 1 & 940.24 & 0.53 & 0.00 & 940.24 & 0.50 & 0.00 \\
\hline
\end{tabular}


Table 4: Results on instances with 25 customers: Part II

\begin{tabular}{|c|c|c|c|c|c|c|c|}
\hline & & \multicolumn{3}{|c|}{ A-ILP } & \multicolumn{3}{|c|}{ T-ILP } \\
\hline Instance & $\mid \overline{|K|}$ & TD & $\overline{\mathrm{CPU}}$ & GAP(\%) & TD & CPU & GAP(\%) \\
\hline$r 202 \_25 \_25 \_0$ & 1 & 1040.78 & 0.11 & 0.00 & 1040.78 & 0.39 & 0.00 \\
\hline r202_25_25_1 & 1 & 873.25 & 5.23 & 0.00 & 873.25 & 0.52 & 0.00 \\
\hline r202_25_25_2 & 1 & 838.37 & 51.59 & 0.00 & 838.37 & 0.77 & 0.00 \\
\hline r202_25_50_0 & 1 & 988.74 & 0.14 & 0.00 & 988.74 & 0.39 & 0.00 \\
\hline r202_25_50_1 & 1 & 774.23 & 1.16 & 0.00 & 774.23 & 0.75 & 0.00 \\
\hline$r 202 \_25 \_50 \_2$ & 1 & 710.15 & 10.91 & 0.00 & 710.15 & 1.30 & 0.00 \\
\hline$r 202 \_25 \_75 \_0$ & 1 & 1081.92 & 0.13 & 0.00 & 1081.92 & 0.36 & 0.00 \\
\hline$r 202 \_25 \_75 \_1$ & 1 & 957.27 & 0.56 & 0.00 & 957.27 & 0.44 & 0.00 \\
\hline$r 202 \_25 \_75 \_2$ & 1 & 929.94 & 6.42 & 0.00 & 929.94 & 0.84 & 0.00 \\
\hline r202_50_25_0 & 1 & 1119.77 & 0.11 & 0.00 & 1119.77 & 0.36 & 0.00 \\
\hline$r 202 \_50 \_25 \_1$ & 1 & 978.45 & 0.17 & 0.00 & 978.45 & 0.52 & 0.00 \\
\hline$r 202 \_50 \_25 \_2$ & 1 & 931.40 & 0.41 & 0.00 & 931.40 & 0.48 & 0.00 \\
\hline r202_50_50_0 & 1 & 1043.97 & 0.11 & 0.00 & 1043.97 & 0.41 & 0.00 \\
\hline r202_50_50_1 & 1 & 908.72 & 0.24 & 0.00 & 908.72 & 0.47 & 0.00 \\
\hline r202_50_50_2 & 1 & 898.57 & 2.45 & 0.00 & 898.57 & 0.88 & 0.00 \\
\hline r202_50_75_0 & 1 & 1080.52 & 0.11 & 0.00 & 1080.52 & 0.36 & 0.00 \\
\hline r202_50_75_1 & 1 & 905.68 & 0.20 & 0.00 & 905.68 & 0.47 & 0.00 \\
\hline$r 202 \_50 \_75 \_2$ & 1 & 882.81 & 0.47 & 0.00 & 882.81 & 0.83 & 0.00 \\
\hline$r 202 \_75 \_25 \_0$ & 2 & 1124.48 & 0.09 & 0.00 & 1124.48 & 0.44 & 0.00 \\
\hline$r 202 \_75 \_25 \_1$ & 1 & 959.51 & 0.13 & 0.00 & 959.51 & 0.39 & 0.00 \\
\hline$r 202 \_75 \_25 \_2$ & 1 & 959.51 & 0.17 & 0.00 & 959.51 & 0.53 & 0.00 \\
\hline r202_75_50_0 & 2 & 1077.29 & 0.11 & 0.00 & 1077.29 & 0.41 & 0.00 \\
\hline r202_75_50_1 & 1 & 863.14 & 0.09 & 0.00 & 863.14 & 0.41 & 0.00 \\
\hline$r 202 \_75 \_50 \_2$ & 1 & 840.97 & 0.16 & 0.00 & 840.97 & 0.56 & 0.00 \\
\hline$r 202 \_75 \_75 \_0$ & 2 & 1161.85 & 0.14 & 0.00 & 1161.85 & 0.41 & 0.00 \\
\hline$r 202 \_75 \_75 \_1$ & 1 & 979.63 & 0.14 & 0.00 & 979.63 & 0.44 & 0.00 \\
\hline$r 202 \_75 \_75 \_2$ & 1 & 960.76 & 0.17 & 0.00 & 960.76 & 0.47 & 0.00 \\
\hline rc108_25_25_0 & 1 & 1364.73 & 0.16 & 0.00 & 1364.73 & 0.34 & 0.00 \\
\hline rc108_25_25_1 & 1 & 1104.76 & 2.22 & 0.00 & 1104.76 & 0.53 & 0.00 \\
\hline rc108_25_25_2 & 1 & 982.27 & 40.69 & 0.00 & 982.27 & 0.78 & 0.00 \\
\hline rc108_25_50_0 & 1 & 1306.69 & 0.14 & 0.00 & 1306.69 & 0.38 & 0.00 \\
\hline rc108_25_50_1 & 1 & 1041.13 & 3.30 & 0.00 & 1041.13 & 0.69 & 0.00 \\
\hline$r c 108 \_25 \_50 \_2$ & 1 & 871.09 & 16.75 & 0.00 & 871.09 & 0.84 & 0.00 \\
\hline rc108_25_75_0 & 1 & 1334.70 & 0.17 & 0.00 & 1334.70 & 0.36 & 0.00 \\
\hline rc108_25_75_1 & 1 & 1205.54 & 4.47 & 0.00 & 1205.54 & 0.81 & 0.00 \\
\hline rc108_25_75_2 & 1 & 1149.82 & 48.26 & 0.00 & 1149.82 & 0.88 & 0.00 \\
\hline rc108_50_25_0 & 2 & 1493.89 & 0.13 & 0.00 & 1493.89 & 0.42 & 0.00 \\
\hline rc108_50_25_1 & 2 & 1316.91 & 3.22 & 0.00 & 1316.91 & 0.56 & 0.00 \\
\hline rc108_50_25_2 & 2 & 1290.27 & 11.08 & 0.00 & 1290.27 & 0.69 & 0.00 \\
\hline rc108_50_50_0 & 2 & 1401.34 & 0.23 & 0.00 & 1401.34 & 0.42 & 0.00 \\
\hline rc108_50_50_1 & 1 & 1030.03 & 0.36 & 0.00 & 1030.03 & 0.45 & 0.00 \\
\hline rc108_50_50_2 & 1 & 994.76 & 6.06 & 0.00 & 994.76 & 1.38 & 0.00 \\
\hline rc108_50_75_0 & 2 & 1563.45 & 0.11 & 0.00 & 1563.45 & 0.45 & 0.00 \\
\hline rc108_50_75_1 & 2 & 1246.31 & 3.63 & 0.00 & 1246.31 & 0.52 & 0.00 \\
\hline rc108_50_75_2 & 1 & 1146.98 & 4.23 & 0.00 & 1146.98 & 0.78 & 0.00 \\
\hline rc108_75_25_0 & 2 & 1741.50 & 0.11 & 0.00 & 1741.50 & 0.39 & 0.00 \\
\hline rc108_75_25_1 & 2 & 1586.04 & 0.49 & 0.00 & 1586.04 & 0.49 & 0.00 \\
\hline rc108_75_25_2 & 2 & 1536.06 & 6.31 & 0.00 & 1536.06 & 0.55 & 0.00 \\
\hline rc108_75_50_0 & 2 & 1516.28 & 0.13 & 0.00 & 1516.28 & 0.47 & 0.00 \\
\hline rc108_75_50_1 & 2 & 1343.63 & 1.05 & 0.00 & 1343.63 & 0.49 & 0.00 \\
\hline rc108_75_50_2 & 2 & 1240.96 & 0.73 & 0.00 & 1240.96 & 0.61 & 0.00 \\
\hline rc108_75_75_0 & 2 & 1658.87 & 0.13 & 0.00 & 1658.87 & 0.41 & 0.00 \\
\hline rc108_75_75_1 & 2 & 1383.92 & 0.59 & 0.00 & 1383.92 & 0.48 & 0.00 \\
\hline rc108_75_75_2 & 2 & 1379.93 & 3.25 & 0.00 & 1379.93 & 0.75 & 0.00 \\
\hline rc205_25_25_0 & 1 & 1274.65 & 0.23 & 0.00 & 1274.65 & 0.42 & 0.00 \\
\hline rc205_25_25_1 & 1 & 885.27 & 182.46 & 0.00 & 885.27 & 0.72 & 0.00 \\
\hline rc205_25_25_2 & 1 & 781.96 & $\dagger$ & 10.79 & 781.96 & 1.19 & 0.00 \\
\hline rc205_25_50_0 & 1 & 1334.79 & 2.94 & 0.00 & 1334.79 & 0.38 & 0.00 \\
\hline rc205_25_50_1 & 1 & 902.44 & 23.55 & 0.00 & 902.44 & 0.69 & 0.00 \\
\hline rc205_25_50_2 & 1 & 794.91 & $\dagger$ & 1.59 & 794.91 & 1.50 & 0.00 \\
\hline rc205_25_75_0 & 1 & 1370.37 & 0.14 & 0.00 & 1370.37 & 0.36 & 0.00 \\
\hline rc205_25_75_1 & 1 & 1072.39 & 4.45 & 0.00 & 1072.39 & 0.89 & 0.00 \\
\hline rc205_25_75_2 & 1 & 970.22 & 14.45 & 0.00 & 970.22 & 0.75 & 0.00 \\
\hline rc205_50_25_0 & 2 & 1679.02 & 0.14 & 0.00 & 1679.02 & 0.48 & 0.00 \\
\hline rc205_50_25_1 & 2 & 1405.13 & 3.17 & 0.00 & 1405.13 & 0.49 & 0.00 \\
\hline rc205_50_25_2 & 2 & 1359.23 & 70.79 & 0.00 & 1359.23 & 0.91 & 0.00 \\
\hline rc205_50_50_0 & 2 & 1432.56 & 0.22 & 0.00 & 1432.56 & 0.42 & 0.00 \\
\hline rc205_50_50_1 & 1 & $1,012.82$ & 1.20 & 0.00 & $1,012.82$ & 0.52 & 0.00 \\
\hline rc205_50_50_2 & 1 & 977.86 & 22.16 & 0.00 & 977.86 & 0.66 & 0.00 \\
\hline rc205_50_75_0 & 2 & 1463.48 & 0.19 & 0.00 & 1463.48 & 0.44 & 0.00 \\
\hline rc205_50_75_1 & 2 & $1,280.18$ & 1.66 & 0.00 & $1,280.18$ & 0.84 & 0.00 \\
\hline$r c 205 \_50 \_75 \_2$ & 1 & 1183.02 & 0.77 & 0.00 & 1183.02 & 0.83 & 0.00 \\
\hline rc205_75_25_0 & 2 & 1610.07 & 0.13 & 0.00 & 1610.07 & 0.41 & 0.00 \\
\hline rc205_75_25_1 & 2 & 1248.10 & 0.44 & 0.00 & 1248.10 & 0.47 & 0.00 \\
\hline rc205_75_25_2 & 2 & 1156.81 & 0.59 & 0.00 & 1156.81 & 0.61 & 0.00 \\
\hline rc205_75_50_0 & 2 & 1536.81 & 0.17 & 0.00 & 1536.81 & 0.39 & 0.00 \\
\hline rc205_75_50_1 & 2 & 1273.82 & 0.41 & 0.00 & 1273.82 & 0.49 & 0.00 \\
\hline rc205_75_50_2 & 2 & 1170.12 & 2.52 & 0.00 & 1170.12 & 0.86 & 0.00 \\
\hline rc205_75_75_0 & 2 & 1574.34 & 0.09 & 0.00 & 1574.34 & 0.41 & 0.00 \\
\hline rc 205_75_75_1 & 2 & 1258.22 & 0.22 & 0.00 & 1258.22 & 0.53 & 0.00 \\
\hline rc205_75_75_2 & 2 & 1212.17 & 0.44 & 0.00 & 1212.17 & 0.69 & 0.00 \\
\hline Average & & 1005.25 & 128.61 & 0.21 & 1005.25 & 0.60 & 0.00 \\
\hline
\end{tabular}




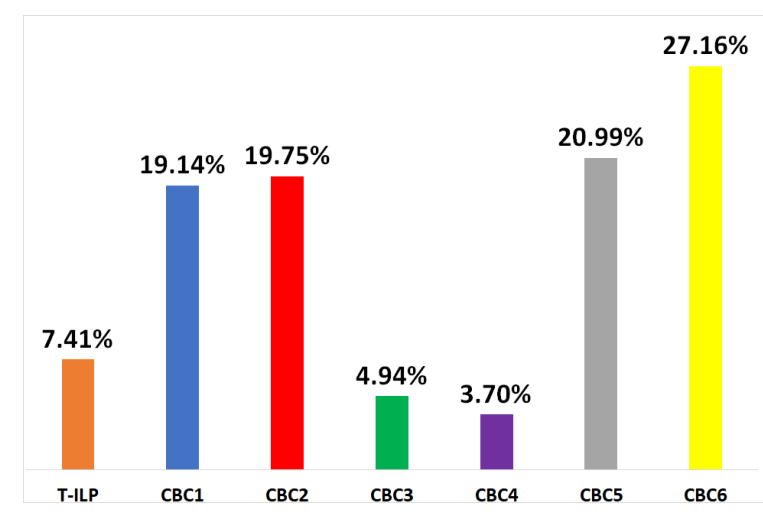

Figure 4: Percentage of instances in which each approach dominates all the others

Tables 7.8 show that on average $\mathrm{CBC} 6$ closes the instances to optimality in an average CPU time that is about $65 \%$ less than that required by the T-ILP model.

In $10 \%$ of the instances, the T-ILP model is not able to find even a feasible solution (cases marked in the tables with the acronym NFS in the TD column) in the time limit of one hour. Indeed, in these cases, the average number of trips generated is more than 1 million. While, on all the instances, the average number of trips is about 242,688 generated in an average CPU time of about 13 seconds. Moreover, in $3 \%$ of the instances, the T-ILP model reaches the CPU time limit of one hour and therefore, it provides only a feasible solution.

Concerning CBC6, in the tables, the cases marked with the symbol ' $*$ ' (about $6 \%$ of the instances) are those in which we report the solution found by the MP at the final iteration, i.e., not a feasible solution for the problem, since the CPU time limit of one hour is reached. CBC6 is able to solve to optimality all instances except two, for which, it provides only infeasible solutions (while the T-ILP model is able to solve them to optimality).

\section{Conclusions and future work}

In this paper, we introduced a new variant of the Containers Drayage Problem (CDP), i.e., the Multi-period Multi-trip CDP with Release and Due Dates (MM-CDP-RDD), in which the planning horizon is divided into discrete time periods (days), each truck can perform more than one trip in a period and customers have to be served in specific periods (Release and Due Dates, RDDs).

We addressed the MM-CDP-RDD through exact approaches. In particular, we proposed an Arc-based Integer Linear Programming (A-ILP) model, representing the problem on a direct graph, properly pre-processed in order to remove infeasible arcs considering both the truck load capacity and 
Table 5: Results on instances with 100 customers: Part I

\begin{tabular}{|c|c|c|c|c|c|c|c|c|c|c|c|c|}
\hline & & \multicolumn{3}{|c|}{ A-ILP } & T-I & $\mathrm{P}$ & $\mathrm{CBC} 1$ & $\mathrm{CBC} 2$ & $\mathrm{CBC} 3$ & $\mathrm{CBC} 4$ & CBC5 & CBC6 \\
\hline 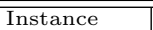 & $\overline{\mid \overline{|K|}}$ & 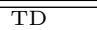 & $\overline{\overline{\mathrm{CPU}}}$ & $\overline{\text { GAP(\%) }}$ & $\overline{T \text { TD }}$ & $\overline{\mathrm{CPU}}$ & 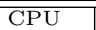 & $\overline{\mathrm{CPU}}$ & $\overline{\mathrm{CPU}}$ & 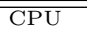 & $\overline{\mathrm{CPU}}$ & CPU \\
\hline c106_25_25_0 & 4 & 3643.51 & $t$ & 18.81 & 3640.19 & 4.06 & 3.47 & 3.16 & 3.03 & 5.38 & 3.28 & 3.27 \\
\hline c106_25_25_1 & 3 & NFS & $\dagger$ & - & 3127.14 & 21.41 & 5.23 & 5.11 & 7.75 & 6.84 & 5.22 & 5.17 \\
\hline c106_25_25_2 & 3 & NFS & $\dagger$ & - & 3018.09 & 89.03 & 78.96 & 78.43 & 128.52 & 814.40 & 29.51 & 12.38 \\
\hline c106_25_50_0 & 3 & NFS & $\dagger$ & - & 3258.41 & 4.25 & 5.06 & 5.33 & 3.34 & 3.13 & 3.59 & 3.14 \\
\hline c106_25_50_1 & 3 & 2846.02 & $\dagger$ & 31.13 & 2656.92 & 31.01 & 5.77 & 5.55 & 14.56 & 6.83 & 5.58 & 7.08 \\
\hline c106_25_50_2 & 3 & NFS & $\dagger$ & - & 2521.93 & 151.91 & $\dagger$ & $\dagger$ & $\dagger$ & $\dagger$ & $\dagger$ & 82.06 \\
\hline c106_25_75_0 & 3 & NFS & $\dagger$ & - & 3462.39 & 4.61 & 3.27 & 3.45 & 3.66 & 5.08 & 3.41 & 4.50 \\
\hline c106_25_75_1 & 3 & 3059.36 & $\dagger$ & 26.27 & 2908.49 & 36.78 & 6.84 & 6.48 & 13.66 & 7.56 & 6.52 & 7.13 \\
\hline c106_25_75_2 & 3 & NFS & $\dagger$ & - & 2791.04 & 201.28 & 49.98 & 50.62 & 280.51 & 442.00 & 37.61 & 25.56 \\
\hline c106_50_25_0 & 4 & 3946.48 & $\dagger$ & 7.93 & 3946.48 & 3.47 & 3.56 & 3.61 & 3.02 & 2.92 & 3.30 & 3.53 \\
\hline c106_50_25_1 & 4 & 3563.41 & $\dagger$ & 23.34 & 3541.00 & 6.27 & 3.70 & 4.20 & 3.67 & 4.03 & 3.59 & 3.17 \\
\hline c106_50_25_2 & 4 & 3452.99 & $\dagger$ & 28.03 & 3437.90 & 22.78 & 5.63 & 5.50 & 6.94 & 5.36 & 5.53 & 5.16 \\
\hline c106_50_50_0 & 4 & 3528.52 & $\dagger$ & 4.90 & 3520.58 & 5.52 & 5.09 & 5.13 & 3.11 & 5.08 & 4.98 & 3.02 \\
\hline c106_50_50_1 & 3 & NFS & $\dagger$ & - & 2879.79 & 47.45 & 4.58 & 4.78 & 6.03 & 5.80 & 4.37 & 3.53 \\
\hline c106_50_50_2 & 3 & NFS & $\dagger$ & - & 2626.06 & 94.40 & 1012.09 & 1005.53 & 1019.07 & $\dagger$ & 244.17 & 367.08 \\
\hline c106_50_75_0 & 4 & 3949.40 & $\dagger$ & 4.80 & 3949.40 & 1.50 & 3.11 & 3.16 & 3.20 & 4.88 & 3.02 & 4.06 \\
\hline c106_50_75_1 & 4 & 3459.67 & $\dagger$ & 10.99 & 3424.65 & 7.53 & 2.92 & 2.84 & 4.77 & 2.81 & 2.59 & 2.72 \\
\hline c106_50_75_2 & 4 & 3427.29 & $\dagger$ & 15.65 & 3375.71 & 30.62 & 6.33 & 6.39 & 15.30 & 8.44 & 6.23 & 6.94 \\
\hline c106_75_25_0 & 5 & 4528.44 & 90.50 & 0.00 & 4528.44 & 0.73 & 0.55 & 0.56 & 0.61 & 0.61 & 0.52 & 0.53 \\
\hline c106_75_25_1 & 4 & 4045.87 & $\dagger$ & 2.87 & 4045.87 & 2.24 & 3.69 & 2.91 & 4.31 & 3.25 & 3.92 & 4.72 \\
\hline c106_75_25_2 & 4 & 3977.54 & $\dagger$ & 8.06 & 3971.76 & 4.28 & 1.73 & 1.44 & 1.67 & 2.89 & 2.66 & 2.64 \\
\hline c106_75_50_0 & 4 & 4011.42 & 43.95 & 0.00 & 4011.42 & 0.81 & 0.59 & 0.56 & 0.66 & 0.59 & 0.53 & 0.58 \\
\hline c106_75_50_1 & 4 & 3493.91 & $\dagger$ & 2.39 & 3493.91 & 3.00 & 0.89 & 2.16 & 1.37 & 2.34 & 3.52 & 0.95 \\
\hline c106_75_50_2 & 4 & 3298.12 & $\dagger$ & 5.51 & 3294.26 & 3.61 & 1.44 & 1.41 & 1.61 & 3.11 & 2.50 & 2.36 \\
\hline c106_75_75_0 & 5 & 4554.96 & 15.906 & 0.00 & 4554.96 & 0.69 & 0.56 & 0.63 & 0.61 & 0.66 & 0.56 & 0.53 \\
\hline c106_75_75_1 & 4 & 4135.05 & $\dagger$ & 1.20 & 4135.05 & 2.92 & 2.89 & 3.08 & 1.20 & 2.38 & 1.05 & 1.99 \\
\hline c106_75_75_2 & 4 & 3977.10 & $\dagger$ & 3.06 & 3977.10 & 3.89 & 1.33 & 1.20 & 1.48 & 2.77 & 1.09 & 3.25 \\
\hline c202_25_25_0 & 4 & 3854.04 & $\dagger$ & 21.49 & 3835.40 & 3.24 & 3.16 & 3.13 & 3.27 & 5.42 & 3.16 & 3.17 \\
\hline c202_25_25_1 & 3 & NFS & $\dagger$ & - & 3392.28 & 22.67 & 4.20 & 4.56 & 5.69 & 4.66 & 4.80 & 4.47 \\
\hline c202_25_25_2 & 3 & NFS & $\dagger$ & - & 3249.86 & 72.39 & 10.87 & 11.00 & 35.47 & 12.91 & 11.03 & 13.28 \\
\hline c202_25_50_0 & 3 & NFS & $\dagger$ & - & 3291.61 & 8.97 & 3.39 & 5.41 & 3.23 & 3.25 & 5.38 & 4.70 \\
\hline c202_25_50_1 & 3 & 2992.99 & $\dagger$ & 33.51 & 2707.84 & 37.45 & 5.80 & 5.61 & 12.20 & 6.58 & 5.75 & 5.34 \\
\hline c202_25_50_2 & 3 & NFS & $\dagger$ & - & 2509.13 & 119.93 & 25.28 & 25.33 & 127.66 & 200.78 & 26.20 & 15.64 \\
\hline c202_25_75_0 & 3 & NFS & $\dagger$ & - & 3421.68 & 4.16 & 3.34 & 3.58 & 3.55 & 3.45 & 3.36 & 3.25 \\
\hline c202_25_75_1 & 3 & 3147.40 & $\dagger$ & 29.06 & 2961.19 & 43.00 & 6.83 & 6.92 & 17.11 & 7.92 & 6.83 & 8.17 \\
\hline c202_25_75_2 & 3 & NFS & $\dagger$ & - & 2842.55 & 183.50 & 24.70 & 24.70 & 196.35 & 28.95 & 24.92 & 27.62 \\
\hline c202_50_25_0 & 4 & 4214.37 & $\dagger$ & 7.88 & 4214.37 & 2.64 & 1.44 & 2.97 & 3.81 & 3.23 & 3.05 & 4.83 \\
\hline c202_50_25_1 & 4 & 3843.97 & $\dagger$ & 22.71 & 3804.34 & 5.23 & 3.44 & 3.49 & 3.56 & 3.22 & 3.61 & 3.45 \\
\hline c202_50_25_2 & 4 & 3769.37 & $\dagger$ & 27.68 & 3689.85 & 21.59 & 5.31 & 5.12 & 9.47 & 6.28 & 5.14 & 4.91 \\
\hline c202_50_50_0 & 4 & 3795.12 & $\dagger$ & 1.36 & 3795.12 & 2.41 & 0.86 & 0.75 & 0.95 & 0.86 & 0.77 & 0.86 \\
\hline c202_50_50_1 & 4 & 3142.52 & $\dagger$ & 8.31 & 3123.99 & 5.66 & 2.36 & 3.75 & 3.50 & 3.72 & 3.45 & 3.37 \\
\hline c202_50_50_2 & 4 & 3108.96 & $\dagger$ & 14.92 & 2976.90 & 16.50 & 4.34 & 4.41 & 7.88 & 4.41 & 4.45 & 3.75 \\
\hline c202_50_75_0 & 4 & 4008.06 & $\dagger$ & 0.82 & 4008.06 & 1.30 & 3.44 & 3.33 & 3.17 & 3.16 & 3.09 & 3.05 \\
\hline c202_50_75_1 & 4 & 3597.99 & $\dagger$ & 8.54 & 3595.81 & 4.45 & 3.41 & 2.36 & 3.17 & 3.61 & 3.58 & 3.20 \\
\hline c202_50_75_2 & 4 & 3642.34 & $\dagger$ & 18.75 & 3533.13 & 18.23 & 4.36 & 5.11 & 9.45 & 5.88 & 4.36 & 4.86 \\
\hline c202_75_25_0 & 5 & 4736.41 & 253.58 & 0.00 & 4736.41 & 0.63 & 0.59 & 0.55 & 0.59 & 0.66 & 0.56 & 0.56 \\
\hline c202_75_25_1 & 5 & 4496.52 & $\dagger$ & 6.65 & 4496.52 & 3.20 & 3.80 & 3.86 & 1.14 & 2.42 & 1.78 & 3.63 \\
\hline c202_75_25_2 & 4 & NFS & $\dagger$ & - & 4376.46 & 5.33 & 2.67 & 1.63 & 1.83 & 2.67 & 2.56 & 2.56 \\
\hline c202_75_50_0 & 4 & 4121.54 & 47.33 & 0.00 & 4121.54 & 0.77 & 0.53 & 0.55 & 0.59 & 0.56 & 0.55 & 0.58 \\
\hline c202_75_50_1 & 4 & 3521.33 & $\dagger$ & 0.95 & 3521.33 & 5.14 & 3.45 & 3.30 & 3.53 & 3.48 & 4.66 & 3.03 \\
\hline c202_75_50_2 & 4 & 3378.33 & $\dagger$ & 2.73 & 3378.33 & 3.28 & 1.50 & 1.59 & 1.59 & 3.19 & 1.78 & 3.28 \\
\hline c202_75_75_0 & 5 & 4669.75 & 10.828 & 0.00 & 4669.75 & 0.64 & 0.59 & 0.61 & 0.66 & 0.63 & 0.63 & 0.55 \\
\hline c202_75_75_1 & 4 & 4256.14 & $\dagger$ & 1.10 & 4256.14 & 3.58 & 1.08 & 1.13 & 5.03 & 5.19 & 1.05 & 1.74 \\
\hline c202_75_75_2 & 4 & 4157.07 & $\dagger$ & 3.59 & 4157.07 & 5.06 & 2.56 & 3.34 & 2.77 & 3.44 & 2.78 & 1.61 \\
\hline r109_25_25_0 & 3 & 3061.91 & $\dagger$ & 16.83 & 3055.01 & 3.31 & 5.05 & 3.30 & 3.30 & 3.00 & 3.27 & 3.17 \\
\hline r109_25_25_1 & 3 & 2854.21 & $\dagger$ & 35.18 & 2707.94 & 15.52 & 4.06 & 3.91 & 5.44 & 4.22 & 4.33 & 4.00 \\
\hline r109_25_25_2 & 3 & 2862.56 & $\dagger$ & 42.25 & 2620.80 & 72.28 & 10.64 & 10.70 & 49.47 & 13.23 & 10.86 & 11.22 \\
\hline r109_25_50_0 & 3 & 2789.39 & $\dagger$ & 18.57 & 2785.85 & 3.34 & 3.25 & 3.36 & 5.16 & 4.86 & 3.25 & 3.08 \\
\hline r109_25_50_1 & 3 & 2582.48 & $\dagger$ & 33.25 & 2425.50 & 40.75 & 8.59 & 8.72 & 17.72 & 9.81 & 9.13 & 8.94 \\
\hline r109_25_50_2 & 3 & NFS & $\dagger$ & - & 2305.31 & 196.32 & 23.06 & 23.05 & 195.17 & 27.53 & 22.91 & 25.70 \\
\hline r109_25_75_0 & 3 & 3058.76 & $\dagger$ & 6.86 & 3058.76 & 5.95 & 2.47 & 5.33 & 5.58 & 5.73 & 5.67 & 5.20 \\
\hline r109_25_75_1 & 3 & 2882.79 & $\dagger$ & 24.31 & 2791.33 & 16.97 & 3.56 & 3.84 & 6.14 & 5.75 & 3.61 & 3.99 \\
\hline r109_25_75_2 & 3 & 3083.84 & $\dagger$ & 37.61 & 2714.56 & 83.95 & 11.84 & 12.13 & 53.09 & 14.45 & 12.05 & 13.73 \\
\hline r109_50_25_0 & 4 & 3477.66 & $\dagger$ & 2.93 & 3477.66 & 2.63 & 4.59 & 3.09 & 2.92 & 3.08 & 5.19 & 2.97 \\
\hline r109_50_25_1 & 4 & 3196.71 & $\dagger$ & 16.21 & 3191.45 & 5.41 & 3.75 & 2.48 & 3.53 & 3.56 & 3.55 & 2.78 \\
\hline r109_50_25_2 & 4 & 3126.26 & $\dagger$ & 20.06 & 3100.93 & 18.62 & 4.17 & 4.28 & 6.16 & 5.56 & 4.03 & 4.31 \\
\hline r109_50_50_0 & 4 & 3053.22 & $\dagger$ & 3.84 & 3053.22 & 2.08 & 2.89 & 3.23 & 3.02 & 2.95 & 3.08 & 2.97 \\
\hline r109_50_50_1 & 4 & 2653.79 & $\dagger$ & 9.77 & 2646.40 & 5.14 & 3.56 & 3.69 & 3.84 & 3.70 & 2.25 & 2.36 \\
\hline r109_50_50_2 & 3 & NFS & $\dagger$ & - & 2492.81 & 37.06 & 518.62 & 517.51 & 522.08 & $\dagger$ & 65.17 & 4.27 \\
\hline r109_50_75_0 & 4 & 3261.69 & $\dagger$ & 5.99 & 3244.50 & 3.17 & 3.48 & 3.06 & 3.28 & 3.27 & 3.08 & 2.95 \\
\hline r109_50_75_1 & 3 & NFS & $\dagger$ & - & 2897.54 & 11.19 & 2.98 & 3.33 & 4.30 & 5.03 & 3.64 & 3.39 \\
\hline r109_50_75_2 & 3 & NFS & $\dagger$ & - & 2833.67 & 41.26 & 6.55 & 6.56 & 19.39 & 8.14 & 6.41 & 6.89 \\
\hline r109_75_25_0 & 4 & 3876.00 & 46.83 & 0.00 & 3876.00 & 0.69 & 0.53 & 0.52 & 0.56 & 0.55 & 0.53 & 0.55 \\
\hline r109_75_25_1 & 4 & 3694.25 & $\dagger$ & 0.35 & 3694.25 & 2.34 & 2.03 & 1.05 & 1.16 & 2.36 & 2.02 & 2.50 \\
\hline r109_75_25_2 & 4 & 3648.58 & $\dagger$ & 8.03 & 3648.58 & 2.84 & 2.27 & 1.39 & 2.05 & 2.72 & 1.27 & 2.39 \\
\hline r109_75_50_0 & 4 & 3466.09 & 4.92 & 0.00 & 3466.09 & 0.69 & 0.63 & 0.59 & 0.58 & 0.63 & 0.58 & 0.56 \\
\hline r109_75_50_1 & 4 & 3023.52 & $\dagger$ & 1.44 & 3023.52 & 3.61 & 2.17 & 1.31 & 1.50 & 6.48 & 2.31 & 3.70 \\
\hline r109_75_50_2 & 4 & 2922.18 & $\dagger$ & 3.44 & 2922.18 & 4.48 & 2.53 & 2.72 & 2.80 & 2.69 & 1.62 & 3.47 \\
\hline r109_75_75_0 & 4 & 3863.58 & 3.38 & 0.00 & 3863.58 & 0.63 & 0.47 & 0.52 & 0.53 & 0.58 & 0.52 & 0.50 \\
\hline r109_75_75_1 & 4 & 3670.58 & 145.25 & 0.00 & 3670.58 & 1.41 & 0.75 & 0.73 & 0.86 & 0.80 & 0.75 & 0.70 \\
\hline r109_75_75_2 & 4 & 3624.69 & $\dagger$ & 1.02 & 3624.69 & 3.11 & 1.59 & 2.63 & 2.73 & 2.66 & 2.67 & 1.24 \\
\hline
\end{tabular}


Table 6: Results on instances with 100 customers: Part II

\begin{tabular}{|c|c|c|c|c|c|c|c|c|c|c|c|c|}
\hline & & & A-ILP & & T-IL & & CBC1 & $\mathrm{CBC} 2$ & CBC3 & $\mathrm{CBC} 4$ & CBC5 & CBC6 \\
\hline Instance & $|K|$ & TD & CPU & GAP(\%) & TD & CPU & CPU & CPU & CPU & CPU & CPU & CPU \\
\hline r207_25_25_0 & 3 & NFS & $t$ & - & 3270.26 & 3.75 & 3.61 & 3.13 & 3.23 & 3.56 & 3.09 & 3.05 \\
\hline r207_25_25_1 & 3 & 3059.99 & $\dagger$ & 41.02 & 2988.41 & 11.50 & 4.08 & 3.66 & 4.23 & 6.38 & 4.30 & 4.36 \\
\hline r207_25_25_2 & 3 & 3053.63 & $\dagger$ & 47.16 & 2889.33 & 30.09 & 5.59 & 5.48 & 10.63 & 6.37 & 5.44 & 5.55 \\
\hline r207_25_50_0 & 3 & 2734.67 & $\dagger$ & 13.79 & 2728.26 & 4.05 & 3.31 & 3.34 & 5.30 & 3.33 & 3.31 & 3.22 \\
\hline r207_25_50_1 & 3 & 2492.90 & $\dagger$ & 29.00 & 2344.16 & 37.83 & 7.53 & 7.28 & 16.16 & 8.89 & 7.53 & 7.64 \\
\hline r207_25_50_2 & 3 & 2608.60 & $\dagger$ & 40.24 & 2199.11 & 254.08 & $\dagger$ & $\dagger$ & & $\dagger$ & $\dagger$ & 114.32 \\
\hline r207_25_75_0 & 3 & 3047.58 & $\dagger$ & 9.46 & 3022.39 & 3.67 & 2.94 & 3.33 & 3.28 & 2.95 & 3.88 & 3.16 \\
\hline r207_25_75_1 & 3 & 2780.16 & $\dagger$ & 23.42 & 2726.13 & 30.90 & 5.66 & 5.84 & 18.33 & 7.81 & 5.72 & 5.88 \\
\hline r207_25_75_2 & 3 & NFS & $\dagger$ & - & 2640.11 & 128.76 & 16.48 & 17.01 & 99.56 & 20.25 & 16.44 & 19.36 \\
\hline r207_50_25_0 & 4 & 3527.80 & $\dagger$ & 9.82 & 3527.80 & 2.77 & 3.03 & 3.31 & 4.92 & 3.05 & 3.09 & 2.95 \\
\hline r207_50_25_1 & 4 & 3190.98 & $\dagger$ & 25.71 & 3179.64 & 5.58 & 2.77 & 3.50 & 3.59 & 4.16 & 2.38 & 3.67 \\
\hline r207_50_25_2 & 4 & 3154.99 & $\dagger$ & 28.32 & 3131.35 & 23.01 & 5.27 & 5.25 & 9.03 & 7.19 & 5.75 & 5.41 \\
\hline r207_50_50_0 & 4 & 3079.74 & $\dagger$ & 4.07 & 3079.74 & 2.91 & 3.19 & 3.41 & 3.28 & 3.25 & 3.17 & 3.06 \\
\hline r207_50_50_1 & 3 & NFS & $\dagger$ & - & 2642.37 & 10.03 & 2.78 & 3.09 & 3.81 & 3.36 & 2.98 & 4.42 \\
\hline r207_50_50_2 & 3 & NFS & $\dagger$ & - & 2455.68 & 58.43 & 1936.14 & 1930.89 & 1939.28 & $\dagger$ & 251.72 & 8.00 \\
\hline r207_50_75_0 & 4 & 3481.45 & $\dagger$ & 2.78 & 3481.45 & 5.11 & 4.33 & 4.83 & 3.17 & 2.97 & 2.97 & 3.00 \\
\hline r207_50_75_1 & 4 & 3265.55 & $\dagger$ & 9.66 & 3265.55 & 4.50 & 2.83 & 2.81 & 2.62 & 3.03 & 3.08 & 2.86 \\
\hline r207_50_75_2 & 4 & 3181.16 & $\dagger$ & 11.75 & 3178.10 & 24.19 & 4.33 & 4.12 & 6.00 & 6.22 & 4.16 & 4.05 \\
\hline r207_75_25_0 & 4 & 3926.16 & 748.126 & 0.00 & 3926.16 & 0.64 & 0.53 & 0.55 & 0.58 & 0.59 & 0.56 & 0.56 \\
\hline r207_75_25_1 & 4 & 3678.48 & $\dagger$ & 1.97 & 3678.48 & 3.59 & 0.78 & 0.89 & 1.00 & 1.02 & 0.84 & 0.84 \\
\hline r207_75_25_2 & 4 & 3615.82 & $\dagger$ & 7.83 & 3615.82 & 4.89 & 2.55 & 2.59 & 2.09 & 3.20 & 2.75 & 2.27 \\
\hline r207_75_50_0 & 4 & 3521.76 & 123.232 & 0.00 & 3521.76 & 2.34 & 2.69 & 2.59 & 2.64 & 2.36 & 2.59 & 2.61 \\
\hline r207_75_50_1 & 4 & 2889.62 & $\dagger$ & 2.02 & 2889.62 & 2.14 & 1.08 & 1.11 & 1.08 & 1.44 & 1.03 & 2.33 \\
\hline r207_75_50_2 & 4 & 2815.52 & $\dagger$ & 4.11 & 2814.72 & 4.67 & 3.39 & 2.45 & 3.66 & 3.44 & 3.58 & 3.14 \\
\hline r207_75_75_0 & 4 & 3691.74 & 16.695 & 0.00 & 3691.74 & 0.73 & 0.56 & 0.59 & 0.66 & 0.58 & 0.58 & 0.56 \\
\hline r207_75_75_1 & 4 & 3399.89 & $\dagger$ & 1.44 & 3399.89 & 3.34 & 1.73 & 4.55 & 4.83 & 4.78 & 5.16 & 1.09 \\
\hline r207_75_75_2 & 4 & 3303.45 & $\dagger$ & 3.24 & 3303.45 & 4.83 & 1.88 & 1.67 & 4.34 & 5.36 & 3.14 & 3.88 \\
\hline rc108_25_25_0 & 4 & 4006.06 & $\dagger$ & 19.20 & 4005.57 & 3.19 & 3.23 & 4.92 & 5.22 & 3.09 & 3.19 & 3.05 \\
\hline rc108_25_25_1 & 4 & NFS & $\dagger$ & - & 3484.63 & 11.66 & 3.83 & 4.08 & 6.06 & 4.23 & 3.92 & 4.27 \\
\hline rc108_25_25_2 & 4 & 3766.30 & $\dagger$ & 44.59 & 3362.70 & 55.79 & 9.20 & 9.16 & 21.22 & 11.03 & 9.20 & 10.06 \\
\hline rc108_25_50_0 & 4 & 3665.40 & $\dagger$ & 12.69 & 3660.40 & 4.70 & 5.41 & 5.50 & 5.23 & 3.37 & 5.53 & 5.20 \\
\hline rc108_25_50_1 & 3 & NFS & $\dagger$ & - & 2947.32 & 32.83 & 5.25 & 5.36 & 20.67 & 6.72 & 5.48 & 5.94 \\
\hline rc108_25_50_2 & 3 & NFS & $\dagger$ & - & 2812.14 & 148.29 & 15.36 & 14.91 & 136.12 & 19.09 & 15.47 & 17.20 \\
\hline rc108_25_75_0 & 4 & 3782.00 & $\dagger$ & 11.49 & 3776.60 & 3.80 & 3.30 & 3.38 & 5.25 & 3.44 & 3.38 & 3.14 \\
\hline rc108_25_75_1 & 3 & NFS & $\dagger$ & - & 3310.69 & 49.12 & 5.77 & 5.73 & 12.27 & 6.95 & 5.61 & 6.94 \\
\hline rc108_25_75_2 & 3 & NFS & $\dagger$ & - & 3156.96 & 160.88 & 324.23 & 328.51 & 552.28 & $\dagger$ & 75.26 & 22.41 \\
\hline rc108_50_25_0 & 4 & 4334.44 & $\dagger$ & 6.11 & 4334.44 & 3.03 & 0.94 & 0.92 & 1.13 & 1.17 & 0.88 & 0.84 \\
\hline rc108_50_25_1 & 4 & 4042.23 & $\dagger$ & 21.12 & 4011.33 & 12.64 & 3.64 & 3.95 & 4.13 & 4.80 & 3.88 & 3.73 \\
\hline rc108_50_25_2 & 4 & 3933.99 & $\dagger$ & 24.85 & 3885.29 & 57.36 & 8.77 & 8.89 & 23.73 & 10.37 & 8.45 & 9.30 \\
\hline rc108_50_50_0 & 4 & 4165.72 & $\dagger$ & 5.14 & 4165.72 & 2.98 & 0.75 & 0.88 & 1.02 & 0.92 & 0.92 & 0.81 \\
\hline rc108_50_50_1 & 4 & 3495.09 & $\dagger$ & 17.76 & 3465.60 & 12.00 & 4.25 & 4.03 & 4.56 & 4.30 & 4.02 & 3.94 \\
\hline rc108_50_50_2 & 4 & 3442.89 & $\dagger$ & 24.29 & 3292.93 & 50.29 & 7.06 & 7.17 & 21.92 & 9.33 & 7.39 & 7.73 \\
\hline rc108_50_75_0 & 4 & 4770.45 & $\dagger$ & 0.60 & 4770.45 & 1.17 & 5.42 & 4.74 & 4.33 & 4.48 & 4.12 & 3.97 \\
\hline rc108_50_75_1 & 4 & 4338.01 & $\dagger$ & 7.85 & 4338.01 & 4.59 & 3.36 & 3.09 & 3.37 & 3.70 & 3.48 & 2.92 \\
\hline rc108_50_75_2 & 4 & 4292.27 & $\dagger$ & 11.97 & 4292.27 & 12.81 & 2.97 & 3.14 & 3.80 & 4.53 & 2.81 & 5.72 \\
\hline rc108_75_25_0 & 5 & 5336.06 & 7.819 & 0.00 & 5336.06 & 0.56 & 0.52 & 0.52 & 0.61 & 0.66 & 0.53 & 0.49 \\
\hline rc108_75_25_1 & 5 & 5049.05 & $\dagger$ & 1.93 & 5049.05 & 1.34 & 0.83 & 0.86 & 0.97 & 0.88 & 0.80 & 0.81 \\
\hline rc108_75_25_2 & 5 & 4967.61 & $\dagger$ & 5.22 & 4967.61 & 4.42 & 1.36 & 1.30 & 1.47 & 2.77 & 1.25 & 2.78 \\
\hline rc108_75_50_0 & 5 & 4709.75 & 10.53 & 0.00 & 4709.75 & 0.84 & 1.64 & 0.61 & 0.64 & 0.70 & 0.66 & 0.58 \\
\hline rc108_75_50_1 & 4 & 4179.12 & 780.01 & 0.00 & 4179.12 & 2.84 & 2.56 & 2.34 & 1.59 & 2.56 & 2.64 & 2.08 \\
\hline rc108_75_50_2 & 4 & 3994.11 & $\dagger$ & 2.04 & 3994.11 & 4.95 & 1.58 & 1.42 & 2.03 & 3.81 & 1.39 & 2.38 \\
\hline rc108_75_75_0 & 5 & 5134.52 & 6.946 & 0.00 & 5134.52 & 0.67 & 0.61 & 0.56 & 0.66 & 0.58 & 0.52 & 0.52 \\
\hline rc108_75_75_1 & 5 & 4785.87 & 3593.941 & 0.00 & 4785.87 & 3.34 & 3.58 & 1.03 & 1.30 & 3.67 & 1.02 & 4.59 \\
\hline rc108_75_75_2 & 5 & 4777.81 & $\dagger$ & 3.77 & 4777.81 & 3.34 & 2.78 & 2.48 & 2.14 & 2.58 & 2.56 & 2.66 \\
\hline rc205_25_25_0 & 4 & 3941.90 & $\dagger$ & 31.09 & 3923.66 & 4.02 & 3.48 & 3.13 & 3.42 & 5.23 & 3.50 & 3.19 \\
\hline rc205_25_25_1 & 3 & NFS & $\dagger$ & - & 3435.78 & 25.70 & 6.70 & 5.16 & 14.95 & 6.78 & 5.05 & 6.36 \\
\hline rc205_25_25_2 & 3 & NFS & $\dagger$ & - & 3339.83 & 128.16 & 17.69 & 15.19 & 74.73 & 18.42 & 16.39 & 16.80 \\
\hline rc205_25_50_0 & 3 & NFS & $\dagger$ & - & 3512.72 & 4.45 & 3.48 & 3.39 & 3.20 & 3.33 & 3.25 & 3.64 \\
\hline rc205_25_50_1 & 3 & 3132.04 & $\dagger$ & 36.75 & 2853.17 & 50.22 & 14.78 & 14.95 & 50.06 & 65.56 & 15.56 & 11.14 \\
\hline rc205_25_50_2 & 3 & NFS & $\dagger$ & - & 2622.99 & 266.98 & 32.36 & 33.00 & 598.12 & 42.48 & 33.56 & 36.25 \\
\hline rc205_25_75_0 & 4 & 4329.62 & $\dagger$ & 6.43 & 4328.99 & 4.95 & 3.06 & 3.13 & 3.14 & 3.17 & 2.91 & 2.78 \\
\hline rc205_25_75_1 & 4 & 3899.40 & $\dagger$ & 19.69 & 3894.89 & 8.77 & 3.28 & 3.19 & 3.78 & 4.36 & 4.55 & 3.89 \\
\hline rc205_25_75_2 & 4 & 3851.37 & $\dagger$ & 28.15 & 3787.87 & 40.69 & 7.05 & 7.06 & 17.31 & 8.61 & 6.66 & 7.56 \\
\hline rc205_50_25_0 & 4 & 4554.98 & $\dagger$ & 5.39 & 4554.98 & 2.86 & 3.16 & 3.27 & 4.98 & 3.20 & 3.19 & 2.94 \\
\hline rc205_50_25_1 & 4 & 4202.91 & $\dagger$ & 23.28 & 4198.08 & 4.70 & 3.56 & 3.44 & 3.58 & 3.63 & 5.52 & 2.89 \\
\hline rc205_50_25_2 & 4 & 4184.31 & $\dagger$ & 28.47 & 4139.36 & 15.86 & 4.36 & 4.05 & 5.78 & 5.77 & 4.83 & 4.38 \\
\hline rc205_50_50_0 & 4 & 4011.97 & $\dagger$ & 2.63 & 4011.97 & 2.91 & 0.89 & 1.84 & 2.41 & 1.95 & 0.83 & 2.75 \\
\hline rc205_50_50_1 & 4 & 3406.10 & $\dagger$ & 17.74 & 3399.79 & 9.62 & 3.23 & 3.03 & 5.61 & 3.80 & 3.37 & 3.27 \\
\hline rc205_50_50_2 & 4 & 3385.27 & $\dagger$ & 26.45 & 3202.24 & 48.25 & 6.75 & 6.59 & 25.59 & 8.30 & 6.61 & 7.19 \\
\hline rc205_50_75_0 & 4 & 4601.11 & $\dagger$ & 2.35 & 4601.11 & 2.84 & 5.02 & 3.22 & 5.11 & 4.84 & 4.95 & 3.06 \\
\hline rc205_50_75_1 & 4 & 4234.35 & $\dagger$ & 10.65 & 4234.09 & 5.56 & 2.34 & 3.16 & 3.53 & 3.70 & 2.28 & 5.17 \\
\hline rc205_50_75_2 & 4 & NFS & $\dagger$ & - & 4126.74 & 26.44 & 3.73 & 3.98 & 6.20 & 4.48 & 3.97 & 4.17 \\
\hline rc205_75_25_0 & 5 & 4846.58 & 41.534 & 0.00 & 4846.58 & 0.69 & 0.56 & 0.59 & 0.63 & 0.69 & 0.56 & 0.58 \\
\hline rc205_75_25_1 & 5 & 4393.80 & $\dagger$ & 3.61 & 4393.80 & 3.66 & 4.89 & 5.08 & 3.58 & 3.75 & 3.48 & 0.92 \\
\hline rc205_75_25_2 & 4 & NFS & $\dagger$ & - & 4260.67 & 5.55 & 2.02 & 2.89 & 3.30 & 3.86 & 1.69 & 2.72 \\
\hline rc205_75_50_0 & 4 & 4306.66 & 99.869 & 0.00 & 4306.66 & 0.80 & 0.59 & 0.55 & 0.63 & 0.69 & 0.56 & 0.55 \\
\hline rc205_75_50_1 & 4 & 3795.39 & $\dagger$ & 3.68 & 3795.39 & 3.78 & 1.06 & 1.09 & 1.28 & 1.25 & 1.00 & 1.03 \\
\hline rc205_75_50_2 & 4 & 3707.18 & $\dagger$ & 6.59 & 3706.77 & 5.34 & 1.98 & 1.83 & 3.67 & 2.31 & 5.73 & 1.88 \\
\hline rc205_75_75_0 & 5 & 5019.14 & 13.187 & 0.00 & 5019.14 & 0.64 & 0.63 & 0.55 & 0.63 & 0.70 & 0.56 & 0.59 \\
\hline rc205_75_75_1 & 5 & 4653.22 & $\dagger$ & 1.12 & 4653.22 & 2.66 & 0.97 & 2.28 & 1.17 & 1.33 & 0.98 & 1.19 \\
\hline rc205_75_75_2 & 5 & 4488.86 & $\dagger$ & 3.50 & 4488.86 & 4.09 & 2.67 & 3.63 & 2.53 & 3.25 & 2.50 & 2.38 \\
\hline Average & & & 3171.01 & & 3562.94 & 24.50 & 72.75 & 72.72 & 86.25 & 147.37 & 52.96 & 8.18 \\
\hline
\end{tabular}


Table 7: Results on instances with 200 customers: Part I

\begin{tabular}{|c|c|c|c|c|c|c|}
\hline & & & \\
\hline Instance & $\overline{|| K \mid}$ & TD & $\overline{\mathrm{CPU}}$ & $\overline{\text { GAP }}$ & $\overline{\mathrm{TD}}$ & CPU \\
\hline C1_2_1_25_25_0 & 8 & 11283.51 & 68.31 & 0.00 & 11283.51 & 9.53 \\
\hline C1_2_1_25_25_1 & 8 & 10404.67 & 1556.78 & 0.00 & 10404.67 & 159.46 \\
\hline C1_2_1_25_25_2 & 8 & NFS & $\dagger$ & - & 10200.63 & 646.94 \\
\hline C1_2_1_25_50_0 & 8 & 10734.76 & 129.85 & 0.00 & 10734.76 & 13.39 \\
\hline C1_2_1_25_50_1 & 8 & 9359.18 & 1626.06 & 0.00 & 9359.18 & 178.20 \\
\hline C1_2_1_25_50_2 & 8 & NFS & $\dagger$ & - & $9019.17^{*}$ & $\dagger$ \\
\hline C1_2_1_25_75_0 & 9 & 12196.92 & 69.56 & 0.00 & 12196.92 & 15.20 \\
\hline C1_2_1_25_75_1 & 8 & 11305.77 & 990.47 & 0.00 & 11305.77 & 115.48 \\
\hline C1_2_1_25_75_2 & 8 & 11251.82 & $\dagger$ & 0.02 & 11064.58 & 541.15 \\
\hline C1_2_1_50_25_0 & 11 & 15078.61 & 16.47 & 0.00 & 15078.61 & 3.34 \\
\hline C1_2_1_50_25_1 & 10 & 14404.63 & 138.93 & 0.00 & 14404.63 & 19.72 \\
\hline C1_2_1_50_25_2 & 10 & 14254.11 & 656.03 & 0.00 & 14254.11 & 67.76 \\
\hline C1_2_1_50_50_0 & 10 & 12549.10 & 20.02 & 0.00 & 12549.10 & 4.17 \\
\hline C1_2_1_50_50_1 & 9 & 11251.10 & 297.80 & 0.00 & 11251.10 & 21.30 \\
\hline C1_2_1_50_50_2 & 9 & 11015.23 & 2728.24 & 0.00 & 11015.23 & 96.67 \\
\hline C1_2_1_50_75_0 & 10 & 14533.41 & 39.33 & 0.00 & 14533.41 & 4.78 \\
\hline C1_2_1_50_75_1 & 10 & 13539.88 & 106.49 & 0.00 & 13539.88 & 13.30 \\
\hline C1_2_1_50_75_2 & 10 & 13422.07 & 570.63 & 0.00 & 13422.07 & 48.72 \\
\hline C1_2_1_75_25_0 & 12 & 16011.96 & 4.48 & 0.00 & 16011.96 & 1.83 \\
\hline C1_2_1_75_25_1 & 11 & 15362.15 & 28.78 & 0.00 & 15362.15 & 5.34 \\
\hline C1_2_1_75_25_2 & 11 & 15289.95 & 101.07 & 0.00 & 15289.95 & 9.75 \\
\hline C1_2_1_75_50_0 & 10 & 13187.62 & 9.44 & 0.00 & 13187.62 & 1.95 \\
\hline C1_2_1_75_50_1 & 10 & 11813.52 & 109.62 & 0.00 & 11813.52 & 7.30 \\
\hline C1_2_1_75_50_2 & 10 & 11466.82 & 373.78 & 0.00 & $11464.51^{*}$ & $\dagger$ \\
\hline C1_2_1_75_75_0 & 11 & 14475.00 & 5.41 & 0.00 & 14475.00 & 3.11 \\
\hline C1_2_1_75_75_1 & 11 & 13607.58 & 28.51 & 0.00 & 13607.58 & 7.84 \\
\hline C1_2_1_75_75_2 & 10 & 13288.32 & 1626.34 & 0.00 & 13288.32 & 12.30 \\
\hline C2_2_3_25_25_0 & 8 & 10123.42 & 89.54 & 0.00 & 10123.42 & 13.55 \\
\hline C2_2_3_25_25_1 & 7 & 9423.27 & 3199.97 & 0.00 & 9423.27 & 265.97 \\
\hline C2 $22 \_3 \_25 \_25 \_2$ & 7 & NFS & $\dagger$ & - & 9121.84 & 650.19 \\
\hline C2_2_3_25_50_0 & 7 & 9251.42 & 381.03 & 0.00 & 9251.42 & 22.97 \\
\hline C2_2_3_25_50_1 & 7 & 8209.24 & $\dagger$ & 0.01 & 8161.40 & 250.99 \\
\hline C2_2_3_25_50_2 & 7 & NFS & $\dagger$ & - & $7766.10^{*}$ & $\dagger$ \\
\hline C2_2_3_25_75_0 & 8 & 10450.37 & 88.90 & 0.00 & 10450.37 & 15.16 \\
\hline C2_2_3_25_75_1 & 8 & 9882.38 & 1052.79 & 0.00 & 9882.38 & 137.52 \\
\hline C2_2_3_25_75_2 & 8 & NFS & $\dagger$ & - & 9696.51 & 547.51 \\
\hline C2_2_3_50_25_0 & 10 & 13105.34 & 13.98 & 0.00 & 13105.34 & 4.31 \\
\hline C2_2_3_50_25_1 & 10 & 12296.73 & 156.98 & 0.00 & 12296.73 & 17.73 \\
\hline C2_2_3_50_25_2 & 10 & 12113.05 & 644.92 & 0.00 & 12113.05 & 62.81 \\
\hline C2_2_3_50_50_0 & 9 & 11342.06 & 18.20 & 0.00 & 11342.06 & 5.56 \\
\hline C2_2_3_50_50_1 & 8 & 9755.95 & 3343.90 & 0.00 & 9755.95 & 28.28 \\
\hline C2_2_3_50_50_2 & 8 & NFS & $\dagger$ & - & $9560.23^{*}$ & $\dagger$ \\
\hline C2_2_3_50_75_0 & 9 & 11844.75 & 19.03 & 0.00 & 11844.75 & 5.69 \\
\hline C2_2_3_50_75_1 & 9 & 10969.32 & 159.79 & 0.00 & 10969.32 & 17.87 \\
\hline C2_2_3_50_75_2 & 9 & 10789.53 & 688.15 & 0.00 & 10789.53 & 67.00 \\
\hline C2_2_3_75_25_0 & 11 & 13946.50 & 4.31 & 0.00 & 13946.50 & 1.86 \\
\hline C2_2_3_75_25_1 & 11 & 13374.59 & 18.53 & 0.00 & 13374.59 & 4.42 \\
\hline C2_2_3_75_25_2 & 10 & 13314.91 & 944.55 & 0.00 & 13314.91 & 8.94 \\
\hline C2_2_3_75_50_0 & 10 & 12016.59 & 7.19 & 0.00 & 12016.59 & 2.41 \\
\hline C2_2_3_75_50_1 & 9 & 11084.88 & 566.03 & 0.00 & 11084.88 & 7.56 \\
\hline C2_2_3_75_50_2 & 9 & 10909.36 & 1375.34 & 0.00 & 10909.36 & 18.78 \\
\hline C2_2_3_75_75_0 & 10 & 12865.15 & 6.80 & 0.00 & 12865.15 & 2.30 \\
\hline C2_2_3_75_75_1 & 10 & 11711.98 & 54.23 & 0.00 & 11711.98 & 8.56 \\
\hline C2_2_3_75_75_2 & 10 & 11587.07 & 338.37 & 0.00 & 11587.07 & 29.19 \\
\hline R1_2_5_25_25_0 & 8 & 11399.57 & 132.21 & 0.00 & 11399.57 & 12.14 \\
\hline R1_2_5_25_25_1 & 8 & 10653.12 & 1099.92 & 0.00 & 10653.12 & 134.99 \\
\hline R1_2_5_25_25_2 & 8 & NFS & $\dagger$ & - & 10418.51 & 419.67 \\
\hline R1_2_5_25_50_0 & 8 & 9865.13 & 97.51 & 0.00 & 9865.13 & 21.08 \\
\hline R1_2_5_25_50_1 & 7 & 8599.48 & 1999.45 & 0.00 & 8599.48 & 543.11 \\
\hline R1_2_5_25_50_2 & 7 & NFS & $\dagger$ & - & $8231.49^{*}$ & $\dagger$ \\
\hline R1_2_5_25_75_0 & 8 & 11349.44 & 67.11 & 0.00 & 11349.44 & 11.66 \\
\hline R1_2_5_25_75_1 & 8 & 10402.82 & 1670.65 & 0.00 & 10402.82 & 151.31 \\
\hline R1_2_5_25_75_2 & 8 & NFS & $\dagger$ & - & 10077.01 & 481.90 \\
\hline R1_2_5_50_25_0 & 10 & 13933.99 & 20.41 & 0.00 & 13933.99 & 5.23 \\
\hline R1_2_5_50_25_1 & 10 & 13231.03 & 235.38 & 0.00 & 13231.03 & 23.81 \\
\hline R1_2_5_50_25_2 & 10 & 12930.91 & 973.64 & 0.00 & 12930.91 & 81.61 \\
\hline R1_2_5_50_50_0 & 9 & 11209.97 & 38.11 & 0.00 & 11209.97 & 6.78 \\
\hline R1_2_5_50_50_1 & 8 & 9822.99 & 513.19 & 0.00 & 9822.99 & 40.50 \\
\hline R1_2_5_50_50_2 & 8 & 9612.11 & $\dagger$ & 0.02 & $9445.51^{*}$ & $\dagger$ \\
\hline R1_2_5_50_75_0 & 10 & 12997.89 & 29.87 & 0.00 & 12997.89 & 5.16 \\
\hline R1_2_5_50_75_1 & 10 & 12212.10 & 253.80 & 0.00 & 12212.10 & 25.64 \\
\hline R1_2_5_50_75_2 & 10 & 11950.30 & 1394.59 & 0.00 & 11950.30 & 107.64 \\
\hline R1_2_5_75_25_0 & 11 & 15720.41 & 4.64 & 0.00 & 15720.41 & 2.06 \\
\hline R1_2_5_75_25_1 & 11 & 15167.18 & 29.55 & 0.00 & 15167.18 & 5.13 \\
\hline R1_2_5_75_25_2 & 11 & 15032.09 & 99.67 & 0.00 & 15032.09 & 10.47 \\
\hline R1_2_5_75_50_0 & 10 & 12991.71 & 9.48 & 0.00 & 12991.71 & 1.73 \\
\hline R1_2_5_75_50_1 & 10 & 11672.34 & 37.86 & 0.00 & 11672.34 & 6.19 \\
\hline R1_2_5_75_50_2 & 10 & 11334.87 & 149.29 & 0.00 & 11334.87 & 523.43 \\
\hline R1_2_5_75_75_0 & 11 & 14995.54 & 5.36 & 0.00 & 14995.54 & 1.97 \\
\hline R1_2_5_75_75_1 & 11 & 14439.77 & 41.73 & 0.00 & 14439.77 & 6.89 \\
\hline R1_2_5_75_75_2 & 11 & 14216.79 & 94.00 & 0.00 & 14216.79 & 13.14 \\
\hline
\end{tabular}


Table 8: Results on instances with 200 customers: Part II

\begin{tabular}{|c|c|c|c|c|c|c|}
\hline & & \multicolumn{3}{|c|}{ T-ILP } & \multicolumn{2}{|c|}{ CBC6 } \\
\hline Instance & $\mid \overline{|K|}$ & TD & $\mathrm{CPU}$ & GAP & TD & $\mathrm{CPU}$ \\
\hline R2_2_9_25_25_0 & 10 & 11971.75 & 69.93 & 0.00 & 11971.75 & 9.31 \\
\hline R2_2_9_25_25_1 & 8 & 11121.18 & 713.70 & 0.00 & 11121.18 & 81.33 \\
\hline R2_2_9_25_25_2 & 8 & NFS & $\dagger$ & - & 10895.77 & 335.48 \\
\hline R2_2_9_25_50_0 & 8 & 10199.67 & 97.73 & 0.00 & 10199.67 & 18.11 \\
\hline R2_2_9_25_50_1 & 8 & NFS & $\dagger$ & - & 8818.51 & 197.38 \\
\hline R2_2_9_25_50_2 & 8 & NFS & $\dagger$ & - & $8412.13^{*}$ & $\dagger$ \\
\hline R2_2_9_25_75_0 & 9 & 12104.21 & 73.46 & 0.00 & 12104.21 & 8.81 \\
\hline R2_2_9_25_75_1 & 8 & 11366.36 & 2583.36 & 0.00 & 11366.36 & 90.90 \\
\hline R2_2_9_25_75_2 & 9 & 11388.06 & $\dagger$ & 0.03 & 11117.62 & 393.06 \\
\hline R2_2_9_50_25_0 & 10 & 13733.07 & 19.51 & 0.00 & 13733.07 & 4.55 \\
\hline R2_2_9_50_25_1 & 10 & 12980.70 & 187.96 & 0.00 & 12980.70 & 21.61 \\
\hline R2_2_9_50_25_2 & 10 & 12694.18 & 786.35 & 0.00 & 12694.18 & 74.76 \\
\hline R2_2_9_50_50_0 & 9 & 11550.48 & 31.61 & 0.00 & 11550.48 & 4.69 \\
\hline R2_2_9_50_50_1 & 9 & 10152.71 & 396.81 & 0.00 & 10152.71 & 47.61 \\
\hline R2_2_9_50_50_2 & 9 & 9951.32 & $\dagger$ & 0.01 & $9813.56^{*}$ & $\dagger$ \\
\hline R2_2_9_50_75_0 & 10 & 13670.67 & 20.55 & 0.00 & 13670.67 & 5.22 \\
\hline R2_2_9_50_75_1 & 10 & 12818.61 & 258.38 & 0.00 & 12818.61 & 26.69 \\
\hline R2_2_9_50_75_2 & 10 & 12563.97 & 991.31 & 0.00 & 12563.97 & 100.64 \\
\hline R2_2_9_75_25_0 & 11 & 15528.91 & 23.56 & 0.00 & 15528.91 & 2.45 \\
\hline R2_2_9_75_25_1 & 11 & 14954.69 & 21.87 & 0.00 & 14954.69 & 3.92 \\
\hline R2_2_9_75_25_2 & 11 & 14773.89 & 84.78 & 0.00 & 14773.89 & 9.55 \\
\hline R2_2_9_75_50_0 & 10 & 13056.48 & 7.20 & 0.00 & 13056.48 & 2.17 \\
\hline R2_2_9_75_50_1 & 10 & 11572.47 & 51.67 & 0.00 & 11572.47 & 7.61 \\
\hline R2_2_9_75_50_2 & 10 & 11228.07 & 278.24 & 0.00 & 11228.07 & 856.79 \\
\hline R2_2_9_75_75_0 & 11 & 15776.70 & 82.12 & 0.00 & 15776.70 & 2.53 \\
\hline R2_2_9_75_75_1 & 11 & 15239.62 & 29.06 & 0.00 & 15239.62 & 4.45 \\
\hline R2_2_9_75_75_2 & 11 & 15048.26 & 92.09 & 0.00 & 15048.26 & 9.64 \\
\hline $\mathrm{RC} 1 \_2 \_2 \_25 \_25 \_0$ & 8 & 11465.54 & 116.04 & 0.00 & 11465.54 & 14.50 \\
\hline $\mathrm{RC} 1 \_2 \_2 \_25 \_25 \_1$ & 8 & 10849.64 & 787.62 & 0.00 & 10849.64 & 97.15 \\
\hline RC1_2_2_25_25_2 & 8 & NFS & $\dagger$ & - & 10680.33 & 356.81 \\
\hline RC1_2_2_25_50_0 & 8 & 9873.04 & 115.90 & 0.00 & 9873.04 & 20.28 \\
\hline RC1_2_2_25_50_1 & 7 & 8485.05 & 1914.00 & 0.00 & 8485.05 & 324.25 \\
\hline RC1_2_2_25_50_2 & 7 & NFS & $\dagger$ & - & $8083.75^{*}$ & $\dagger$ \\
\hline RC1_2_2_25_75_0 & 8 & 11407.10 & 72.15 & 0.00 & 11407.10 & 12.67 \\
\hline RC1_2_2_25_75_1 & 8 & 10633.50 & 678.44 & 0.00 & 10633.50 & 95.42 \\
\hline $\mathrm{RC} 1 \_2 \_2 \_25 \_75 \_2$ & 8 & NFS & $\dagger$ & - & 10479.76 & 336.06 \\
\hline RC1_2_2_50_25_0 & 10 & 12685.85 & 21.67 & 0.00 & 12685.85 & 4.75 \\
\hline RC1_2_2_50_25_1 & 9 & 11743.68 & 285.58 & 0.00 & 11743.68 & 31.83 \\
\hline RC1_2_2_50_25_2 & 9 & 11538.25 & 1919.08 & 0.00 & 11538.25 & 139.03 \\
\hline RC1_2_2_50_50_0 & 9 & 11901.84 & 29.67 & 0.00 & 11901.84 & 7.17 \\
\hline RC1_2_2_50_50_1 & 9 & 10389.54 & 360.47 & 0.00 & 10389.54 & 46.42 \\
\hline RC1_2_2_50_50_2 & 9 & 10179.69 & 2638.32 & 0.00 & 10179.69 & 276.22 \\
\hline RC1_2_2_50_75_0 & 10 & 14026.09 & 17.22 & 0.00 & 14026.09 & 5.77 \\
\hline RC1_2_2_50_75_1 & 10 & 13322.63 & 111.95 & 0.00 & 13322.63 & 15.36 \\
\hline RC1_2_2_50_75_2 & 10 & 13159.21 & 611.49 & 0.00 & 13159.21 & 58.25 \\
\hline $\mathrm{RC} 1 \_2 \_2 \_75 \_25 \_0$ & 12 & 15790.92 & 4.22 & 0.00 & 15790.92 & 2.14 \\
\hline RC1_2_2_75_25_1 & 11 & 15473.16 & 68.83 & 0.00 & 15473.16 & 4.22 \\
\hline RC1_2_2_75_25_2 & 11 & 15315.91 & 81.53 & 0.00 & 15315.91 & 8.30 \\
\hline RC1_2_2_75_50_0 & 10 & 12801.00 & 4.84 & 0.00 & 12801.00 & 1.73 \\
\hline RC1_2_2_75_50_1 & 10 & 11439.60 & 27.77 & 0.00 & 11439.60 & 5.42 \\
\hline $\mathrm{RC} 1 \_2 \_2 \_75 \_50 \_2$ & 10 & 11147.26 & 120.09 & 0.00 & 11147.26 & 10.66 \\
\hline $\mathrm{RC} 1 \_2 \_2 \_75 \_75 \_0$ & 11 & 15241.89 & 4.20 & 0.00 & 15241.89 & 2.20 \\
\hline RC1_2_2_75_75_1 & 11 & 14493.83 & 28.61 & 0.00 & 14493.83 & 4.72 \\
\hline RC1_2_2_75_75_2 & 11 & 14408.80 & 134.99 & 0.00 & 14408.80 & 11.36 \\
\hline $\mathrm{RC} 2 \_2 \_2 \_25 \_25 \_0$ & 9 & 11996.43 & 67.72 & 0.00 & 11996.43 & 10.38 \\
\hline RC2 $22 \_2 \_25 \_25 \_1$ & 8 & 11112.52 & 567.46 & 0.00 & 11112.52 & 68.40 \\
\hline RC2 $2 \_2 \_25$-25_2 & 8 & 10880.95 & 2566.52 & 0.00 & 10880.95 & 257.74 \\
\hline RC2 $22 \_2 \_25 \_50 \_0$ & 8 & 10288.10 & 81.67 & 0.00 & 10288.10 & 11.61 \\
\hline RC2_2_2_25_50_1 & 8 & 9049.90 & 1290.97 & 0.00 & 9049.90 & 166.18 \\
\hline RC2 $22 \_2 \_25 \_50 \_2$ & 8 & NFS & $\dagger$ & - & 8768.07 & 665.42 \\
\hline RC2 $22 \_2 \_25 \_75 \_0$ & 9 & 12390.03 & 38.92 & 0.00 & 12390.03 & 8.12 \\
\hline RC2 $22 \_2 \_25 \_75 \_1$ & 9 & 11702.26 & 589.61 & 0.00 & 11702.26 & 59.51 \\
\hline RC2_2_2_25_75_2 & 9 & 11554.80 & 2161.88 & 0.00 & 11554.80 & 201.46 \\
\hline RC2 $22 \_2 \_50 \_25 \_0$ & 10 & 13935.33 & 21.01 & 0.00 & 13935.33 & 4.89 \\
\hline $\mathrm{RC} 2 \_2 \_2 \_50 \_25 \_1$ & 10 & 13157.47 & 203.38 & 0.00 & 13157.47 & 22.00 \\
\hline RC2 $22 \_2 \_50 \_25 \_2$ & 10 & 13011.45 & 715.73 & 0.00 & 13011.45 & 73.20 \\
\hline RC2_2_2_50_50_0 & 9 & 11753.09 & 30.14 & 0.00 & 11753.09 & 5.89 \\
\hline RC2_2_2_50_50_1 & 8 & 9687.19 & 505.96 & 0.00 & 9687.19 & 56.43 \\
\hline RC2_2_2_50_50_2 & 8 & 9382.32 & $\dagger$ & 0.01 & 9271.31 & 197.02 \\
\hline RC2 $22 \_2 \_50 \_75 \_0$ & 10 & 13083.61 & 23.69 & 0.00 & 13083.61 & 4.94 \\
\hline RC2_2_2_50_75_1 & 9 & 12309.00 & 294.35 & 0.00 & 12309.00 & 26.09 \\
\hline RC2_2_2_50_75_2 & 9 & 12129.82 & 1103.73 & 0.00 & 12129.82 & 97.15 \\
\hline RC2 $22 \_2 \_75 \_25 \_0$ & 11 & 14765.00 & 6.45 & 0.00 & 14765.00 & 1.83 \\
\hline RC2 $22 \_2 \_75 \_25 \_1$ & 11 & 13917.72 & 40.97 & 0.00 & 13917.72 & 7.78 \\
\hline RC2 $22 \_2 \_75 \_25 \_2$ & 11 & 13700.43 & 157.77 & 0.00 & 13700.43 & 17.45 \\
\hline RC2 $22 \_2 \_75 \_50 \_0$ & 11 & 13466.10 & 5.56 & 0.00 & 13466.10 & 1.81 \\
\hline RC2_2_2_75_50_1 & 10 & 11406.55 & 35.67 & 0.00 & 11406.55 & 6.22 \\
\hline RC2 $22 \_2 \_75 \_50 \_2$ & 10 & 10803.64 & 733.54 & 0.00 & $10803.36^{*}$ & $\dagger$ \\
\hline $\mathrm{RC} 2 \_2 \_2 \_75 \_75 \_0$ & 12 & 16883.64 & 3.34 & 0.00 & 16883.64 & 1.69 \\
\hline RC2_2_2_75_75_1 & 12 & 16476.26 & 18.69 & 0.00 & 16476.26 & 3.42 \\
\hline RC2 $22 \_2 \_75 \_75 \_2$ & 12 & 16440.09 & 65.61 & 0.00 & 16440.09 & 7.77 \\
\hline Average & & & 870.73 & & & 302.75 \\
\hline
\end{tabular}


the RDDs. However, due to both the maximum truck load capacity and the RDDs, the number of feasible trips may be very limited. Therefore, we also designed a Trip-based solution approach where all the feasible nondominated trips are firstly determined and then, a Trip-based ILP (T-ILP) model is solved. Moreover, because the number of feasible non-dominated trips may become high on medium/large-sized instances, we also proposed six different Combinatorial Benders' Cuts (CBC) approaches for efficiently solving T-ILP model, by defining, beyond the traditional CBC, ad hoc both valid inequalities and stronger cuts.

The proposed approaches were tested on 486 instances, with different number of customers $(25,100$ and 200), derived from those proposed for the VRP. On 25-customers instances, T-ILP model outperformed all the other approaches including A-ILP model that reached the CPU time limit in 4 cases. On 100-customers instances, on average, the sixth CBC method outperformed the others, although it is shown that, instance by instance, it did not always dominate the others. Finally, on the instances with 200 customers, the sixth CBC method strongly outperformed the T-ILP model closing to optimality almost all the instances in an average CPU time that is about $65 \%$ less than that required by the T-ILP model (302.75 vs 870.73 seconds).

\section{References}

[1] S. Akpinar, A. Elmi, and T. Bektaş. Combinatorial benders cuts for assembly line balancing problems with setups. European Journal of Operational Research, 259(2):527-537, 2017.

[2] C. Archetti, O. Jabali, and M. G. Speranza. Multi-period vehicle routing problem with due dates. Computers \& Operations Research, 61:122$134,2015$.

[3] L. Bai and P A. Rubin. Combinatorial benders cuts for the minimum tollbooth problem. Operations Research, 57:1510-1522, 2009.

[4] J. Benders. Partitioning procedures for solving mixed-variables programming problems. Numerische Mathematik, 4:238-252, 1962.

[5] K. Braekers, A. Caris, and G. K. Janssens. Integrated planning of loaded and empty container movements. OR spectrum, 35(2):457-478, 2013.

[6] K. Braekers, A. Caris, and G. K. Janssens. Bi-objective optimization of drayage operations in the service area of intermodal terminals. Transportation Research Part E: Logistics and Transportation Review, 65:50-69, 2014. 
[7] C. Caballini, I. Rebecchi, and S. Sacone. Combining multiple trips in a port environment for empty movements minimization. Transportation Research Procedia, 10:694-703, 2015.

[8] J.X. Cao, D-H. Lee, J.H. Chen, and Q. Shi. The integrated yard truck and yard crane scheduling problem: Benders decomposition-based methods. Transportation Research Part E: Logistics and Transportation Review, 46(3):344-353, 2010.

[9] A. Caris and G. K. Janssens. A local search heuristic for the pre-and end-haulage of intermodal container terminals. Computers \& Operations Research, 36(10):2763-2772, 2009.

[10] J.H. Chen, D-H. Lee, and J.X. Cao. A combinatorial benders cuts algorithm for the quayside operation problem at container terminals. Transportation Research Part E: Logistics and Transportation Review, 48(1):266-275, 2012.

[11] K. H. Chung, C. S. Ko, J. Y. Shin, H. Hwang, and K. H. Kim. Development of mathematical models for the container road transportation in korean trucking industries. Computers $8 \mathcal{G}$ Industrial Engineering, 53(2):252-262, 2007.

[12] G. Codato and M. Fischetti. Combinatorial benders' cuts for mixedinteger linear programming. Operations Research, 54:756-766, 2006.

[13] J-F. Côté, M. Dell'Amico, and M. Iori. Combinatorial benders' cuts for the strip packing problem. Operations Research, 62(3):643-661, 2014.

[14] P. Francis, G. Zhang, and K. Smilowitz. Improved modeling and solution methods for the multi-resource routing problem. European Journal of Operational Research, 180(3):1045-1059, 2007.

[15] A. Geoffrion. Generalized benders decomposition. Journal of Optimization Theory and Applications, 10:237-260, 1972.

[16] A. Ghezelsoflu, M. Di Francesco, A. Frangioni, and P. Zuddas. A setcovering formulation for a drayage problem with single and double container loads. Journal of Industrial Engineering International, pages $1-12,2018$.

[17] M. Gronalt, R. F. Hartl, and M. Reimann. New savings based algorithms for time constrained pickup and delivery of full truckloads. European Journal of Operational Research, 151(3):520-535, 2003.

[18] Jörg Homberger and Hermann Gehring. A two-phase hybrid metaheuristic for the vehicle routing problem with time windows. European Journal of Operational Research, 162(1):220-238, 2005. 
[19] J. Hooker and G. Ottoson. Logic-based benders decomposition. Mathematical Programming, 96:33-60, 2003.

[20] A. Imai, E. Nishimura, and J. Current. A lagrangian relaxation-based heuristic for the vehicle routing with full container load. European journal of operational research, 176(1):87-105, 2007.

[21] H. Jula, M. Dessouky, P. Ioannou, and A. Chassiakos. Container movement by trucks in metropolitan networks: modeling and optimization. Transportation Research Part E: Logistics and Transportation Review, 41(3):235-259, 2005.

[22] M. Lai, M. Battarra, M. Di Francesco, and P. Zuddas. An adaptive guidance meta-heuristic for the vehicle routing problem with splits and clustered backhauls. Journal of the Operational Research Society, 66(7):1222-1235, 2015.

[23] M. Lai, T. G. Crainic, M. Di Francesco, and P. Zuddas. An heuristic search for the routing of heterogeneous trucks with single and double container loads. Transportation Research Part E: Logistics and Transportation Review, 56:108-118, 2013.

[24] Gonzalez-Feliu J. Mancini, S. A mip formulation for a combined vehicle routing and driver scheduling problem with real life constraints. pages 760-773, 2014.

[25] S. Mancini, M. Ciavotta, and C. Meloni. The multiple multidimensional knapsack with family-split penalties. European Journal of Operational Research, in press, 2019.

[26] N. Marković, Ž. Drobnjak, and P. Schonfeld. Dispatching trucks for drayage operations. Transportation Research Part E: Logistics and Transportation Review, 70:99-111, 2014.

[27] P. Nagl. Longer combination vehicles (lcv) for asia and pacific region: Some economic implications. UNESCAP Working Papers n2, UNITED NATIONS, 2007.

[28] R. Namboothiri and A. L. Erera. Planning local container drayage operations given a port access appointment system. Transportation Research Part E: Logistics and Transportation Review, 44(2):185-202, 2008 .

[29] J. Nossack and E. Pesch. A truck scheduling problem arising in intermodal container transportation. European Journal of Operational Research, 230(3):666-680, 2013. 
[30] S. N. Parragh, K. F. Doerner, and R. F. Hartl. A survey on pickup and delivery problems. Journal für Betriebswirtschaft, 58(1):21-51, 2008.

[31] Sophie N Parragh, Karl F Doerner, and Richard F Hartl. A survey on pickup and delivery models part ii: Transportation between pickup and delivery locations. Journal für Betriebswirtschaft, 58(2):81-117, 2008.

[32] L. B. Reinhardt, D. Pisinger, S. Spoorendonk, and M. M. Sigurd. Optimization of the drayage problem using exact methods. INFOR: Information Systems and Operational Research, 54(1):33-51, 2016.

[33] F. Schulte, E. Lalla-Ruiz, R. G. González-Ramírez, and S. Voß. Reducing port-related empty truck emissions: a mathematical approach for truck appointments with collaboration. Transportation Research Part E: Logistics and Transportation Review, 105:195-212, 2017.

[34] S. Shiri and N. Huynh. Optimization of drayage operations with timewindow constraints. International Journal of Production Economics, 176:7-20, 2016.

[35] S. Shiri and N. Huynh. Assessment of us chassis supply models on drayage productivity and air emissions. Transportation Research Part D: Transport and Environment, 61:174-203, 2018.

[36] Marius M Solomon. Algorithms for the vehicle routing and scheduling problems with time window constraints. Operations research, 35(2):254-265, 1987.

[37] D. W. Song and P. Panayides. Maritime logistics: A complete guide to effective shipping and port management. Kogan Page Publishers, 2012.

[38] Y. Song, J. Zhang, Z. Liang, and C. Ye. An exact algorithm for the container drayage problem under a separation mode. Transportation Research Part E: Logistics and Transportation Review, 106:231-254, 2017.

[39] S. Sterzik and H. Kopfer. A tabu search heuristic for the inland container transportation problem. Computers $\&$ Operations Research, 40(4):953-962, 2013.

[40] Z.C. Taşkin and M. Cevik. Combinatorial benders cuts for decomposing imrt fluence maps using rectangular apertures. Computers $\mathscr{E}$ Operations Research, 40(9):2178-2186, 2013.

[41] J. Verstichel, J. Kinable, P. De Causmaecker, and G. Vanden Berghe. A combinatorial benders decomposition for the lock scheduling problem. Computers $\& 3$ Operations Research, 54:117-128, 2015. 
[42] M. Vidović, M. Nikolić, and D. Popović. Two mathematical formulations for the containers drayage problem with time windows. International Journal of Business Science and Applied Management, 7(3):2332, 2012.

[43] M. Vidović, D. Popović, B. Ratković, and G. Radivojević. Generalized mixed integer and vns heuristic approach to solving the multisize containers drayage problem. International Transactions in Operational Research, 24(3):583-614, 2017.

[44] M. Vidović, G. Radivojević, and B. Raković. Vehicle routing in containers pickup up and delivery processes. Procedia-Social and Behavioral Sciences, 20:335-343, 2011.

[45] W. F. Wang and W. Y. Yun. Scheduling for inland container truck and train transportation. International journal of production economics, 143(2):349-356, 2013.

[46] X. Wang and A. C. Regan. Local truckload pickup and delivery with hard time window constraints. Transportation Research Part B: Methodological, 36(2):97-112, 2002.

[47] Z. Xue, W.H. Lin, L. Miao, and C. Zhang. Local container drayage problem with tractor and trailer operating in separable mode. Flexible Services and Manufacturing Journal, 27(2-3):431-450, 2015.

[48] Z. Xue, C. Zhang, W.H. Lin, L. Miao, and P. Yang. A tabu search heuristic for the local container drayage problem under a new operation mode. Transportation Research Part E: Logistics and Transportation Review, 62:136-150, 2014.

[49] R. Zhang, W. Y. Yun, and H. Kopfer. Heuristic-based truck scheduling for inland container transportation. OR spectrum, 32(3):787-808, 2010.

[50] R. Zhang, W. Y. Yun, and I. Moon. A reactive tabu search algorithm for the multi-depot container truck transportation problem. Transportation Research Part E: Logistics and Transportation Review, 45(6):904-914, 2009 .

[51] R. Zhang, W. Y. Yun, and I. Moon. Modeling and optimization of a container drayage problem with resource constraints. International Journal of Production Economics, 133(1):351-359, 2011. 